\title{
An adaptive reference/test paradigm: Application to pulsed fluoroscopy perception
}

\author{
PING XUE, CECIL W. THOMAS, GROVER C. GILMORE, and DAVID L. WILSON \\ Case Western Reserve University, Cleveland, Ohio
}

\begin{abstract}
We developed an adaptive forced-choice method whereby reference and test presentations were alternated in order to minimize effects from variables such as subject attention level. In our demonstration example of an X-ray fluoroscopy perception study, we measured detectability of low-contrast objects in noisy image sequences and determined $\mathrm{X}$-ray dose levels for equivalent detectability of identical contrasts for a new test acquisition method (fluoroscopy at $15 \mathrm{acq} / \mathrm{sec}$ ) as compared with a reference (conventional fluoroscopy at $30 \mathrm{acq} / \mathrm{sec}$ ). In preliminary experiments, we found a dose savings with the test method. We derived parameter uncertainties for the adaptive procedure and demonstrated their applicability with Monte Carlo simulations. Repeated experiments on a single subject demonstrated reduced standard errors due to the reduction of day-to-day variations. It is believed that the method can be applied in a variety of situations in which one needs to compare perception measurements.
\end{abstract}

We have developed a new experimental method that addresses issues in human perception studies. First, a subject's response can vary due to fatigue, a lapse in attention, or possible physiological changes. Kahneman (1973) discussed the importance of attention in perception experiments, indicating that attention may affect the sensory mechanism as well as the decision mechanism. Taylor (1967) observed variation in performance from one day to the next, and others (Hall, 1981; Madigan \& Williams, 1987) reported variations within single experiments. This variation complicates comparison of results. For example, in our experiments in medical X-ray fluoroscopy, we compared new test acquisition methods with an established reference technique. Some of the effects that we wished to measure are comparable to day-to-day variations, and our solution was to alternate reference and test presentations over a time scale of seconds and obtain responses under identical conditions. Second, variability between subjects also presents a dilemma. For an independent variable such as image contrast, many trial-and-error presentations are required to arrive at display conditions that match the floor and ceiling responses of all subjects. This problem is well known, and adaptive techniques can solve the problem (Findlay, 1978; Hall, 1981; King-Smith, Grigsby, Vingrys, Benes, \& Supowit, 1994; Pentland, 1980; Simpson, 1989; Taylor, 1967; Wat-

This work was supported by the Whitaker Foundation and NIH Grant R29-HL48918. We thank Richard Aufrichtig for many helpful suggestions, Arthur Burgess for suggesting that we use adaptive methods, and the reviewers for their useful comments. P.X. is in the Department of Biomedical Engineering, Case Western Reserve University; C.W.T. is in the Departments of Biomedical Engineering and Psychology at Case Western Reserve University; G.C.G. is in the Department of Psychology, Case Western Reserve University; and D.L.W. is in the Departments of Biomedical Engineering and Radiology, Case Western Reserve University. Correspondence should be addressed to D. L. Wilson, Department of Biomedical Engineering, Case Western Reserve University, Cleveland, $\mathrm{OH} 44106$ (e-mail: dlw(a)po.cwru.edu). son \& Pelli, 1983). Third, one desires an objective experimental method such as a forced-choice technique (Blackwell, 1963; Elliott, 1964; Green \& Swets, 1966; Ohara, Doi, Metz, \& Giger, 1989). In the present paper, we address all of these issues and more using a new, interspersed reference/test, adaptive forced-choice method with $M$ alternatives.

The method is demonstrated using an application in medical imaging perception. However, we believe that the method is generally applicable, and potential additional applications in image perception are described in the Discussion section.

\section{Fluoroscopy Perception Experiments}

In fluoroscopy, it is desirable to minimize X-ray dose to the patient while achieving an acceptable level of object detectability. Noise is due to statistical fluctuations in the number of detected X-ray photons. Increasing the $\mathrm{X}$-ray dose reduces image noise and improves object detection at the expense of increasing X-ray exposure to patients and staff. Reducing radiation dose makes the images noisier and reduces detectability of objects in the image. A change in dose is normally obtained by changing the current to the X-ray tube, and this does not affect image contrast.

We studied pulsed fluoroscopy at reduced image acquisition rates, an imaging technique that may reduce $\mathrm{X}$-ray dose to patients and staff, as reviewed elsewhere (Aufrichtig, Xue, Thomas, Gilmore, \& Wilson, 1994). In pulsed fluoroscopy, X-rays are delivered in short pulses of radiation (typically $0.2-3.0 \mathrm{msec}$ ) that are in synchrony with video image recording and display. Pulsed fluoroscopy images may be recorded at $30 \mathrm{acq} / \mathrm{sec}$ (acquisitions per second). Since conventional video requires 30 frames per second, each acquisition frame becomes one video frame in a 30-frames/sec display. We refer to this as "pulsed-30." Pulsed fluoroscopy images are also acquired at reduced 
rates, typically $15 \mathrm{acq} / \mathrm{sec}$, or pulsed- 15 . To conform to the standard 30 -frames/sec video display, each acquisition is displayed twice. Pulsed-10 and pulsed-7.5 are other possibilities.

Previously, we performed human perception experiments to determine proper X-ray dose levels for pulsed fluoroscopy at reduced acquisition rates (Aufrichtig, Xue, et al., 1994). In computer-generated, continuously repeated (cine) displays of low-contrast disks in the noisy images, we varied the dose per acquisition of pulsed-15 in order to find a level that gave disk detectability equivalent to pulsed-30. An operationally defined equivalent perception dose (EPD) was obtained using three different experimental paradigms: paired comparison, minimum contrast, and forced choice (Aufrichtig, Xue, et al., 1994). Using EPD values and the acquisition rate, we computed dose savings over a time interval. X-ray dose savings depended upon disk size and acquisition rate, and the average dose savings of pulsed- 15 was approximately $22 \%$. Data were well described by a human observer model (Aufrichtig, Thomas, Xue, \& Wilson, 1994) that included the spatiotemporal response of the human visual system measured by Kelly $(1979,1984)$.

The fluoroscopy experiments are technically demanding because each presentation consists of a sequence of 100 frames, each containing a unique noise realization. In earlier experiments, we emphasized paired-comparison and minimum-contrast techniques because we were able to include more disk sizes on a single display and save experiment time as compared with the forced-choice technique (Aufrichtig, Xue, et al., 1994). Nevertheless, we concluded in this paper and elsewhere (Wilson, Xue, \& Aufrichtig, 1994b) that forced choice was desirable because it avoided inter- and intraobserver differences in the threshold criterion and measured a response at lower contrasts. Similar observations were made years ago by Blackwell (1963). Moreover, others using forced choice exclusively reported smaller dose savings (Whiting, Eckstein, \& Eigler, 1994). New computers with enhanced video capabilities and large, fast memories allow us to more easily perform experiments on sequences of images. For these reasons, we decided to revisit forced-choice experiments.

Our ultimate goal is to minimize $\mathrm{X}$-ray dose utilization in clinical practice by optimizing acquisition and filtering techniques. A decreased X-ray dose per second leads to a dose savings, while using fluoroscopy for longer durations increases $\mathrm{X}$-ray dose. It is important to assess both factors, and one attempt at the latter is response time measurements. In experiments, we do not want to measure how long a subject must take to detect a target; instead, we want to determine how long a subject will take in a free response situation that more closely mimics clinical practice. Hence, in experiments, we measure the unrestricted, response-terminated time. This experiment is suitable for our purposes. However, if one is interested in isolating and studying the properties of a percept response time that is free of decisional factors, one might want to use a different paradigm such as a measurement of the speed-accuracy tradeoff function, as described in the Discussion section.

\section{Overview}

In Figure 1, we chart the experimental variables in our experiment. Variable names (first column) include the desired probability correct, the image acquisition rate, the X-ray dose (which, as described later, is related to the quantum noise level), and object contrast. In the case of the reference condition (second column), the acquisition rate is $30 \mathrm{acq} / \mathrm{sec}$, and dose is fixed at a constant level that mimics conventional fluoroscopy. Contrast is adapted to achieve $80 \%$ probability of correct responses, and this ensures that presentations are individualized to a subject's floor and ceiling response and that optimal statistics are obtained (see Results section). In the case of the test condition (third column), the acquisition rate is reduced (e.g., $15 \mathrm{acq} / \mathrm{sec}$ ). The contrast of test is set equal to that in reference in order to mimic the physics of fluoroscopy instrumentation, as described previously. This linkage between the reference and test conditions is indicated by the arrow in the figure. In the case of test, the dose (noise) level is adapted to get $80 \%$ correct. As a result of the linkage, the adapted dose for test is the dose for equivalent detectability of a contrast identical to that used in reference. At the bottom of the figure, outputs are explicitly listed. The final value of the dose is the EPD, the principal experimental output. Other outputs are absolute detectabilities, contrast, and subject response times.

In this report, details of the new method are described. Because noisy image sequences are used, significant technical challenges must be overcome. We derived parameter uncertainties and evaluated them with Monte Carlo simulations. The technique is demonstrated with EPD experiments. Finally, we repeated experiments several times on a single subject in order to compare measured, theoretical, and Monte Carlo variances.

\begin{tabular}{|l|l|l|}
\hline \multicolumn{1}{|c|}{ Variables } & Reference & \multicolumn{1}{c|}{ Test } \\
\hline probability correct & $80 \%$ & $80 \%$ \\
acquisition rate & $30 \mathrm{acq} / \mathrm{sec}$ & $15 \mathrm{acq} / \mathrm{sec}$ \\
dose (noise) & constant & adapted \\
contrast & adapted & linked \\
\hline
\end{tabular}

\begin{tabular}{|l|l|}
\hline Outputs & $\begin{array}{l}\text { EPD (dose of test) } \\
\text { absolute detectabilities } \\
\text { contrast } \\
\text { response times }\end{array}$ \\
\hline
\end{tabular}

Figure 1. The basic experimental paradigm for a reference/test equivalent perception dose (EPD) experiment. Experimental variables are dose (which determines the noise level), object contrast, acquisition rate, and the desired probability correct. As indicated by the arrow, the contrast in the test presentation is linked to that in the reference presentation. 


\section{Adaptive Forced-Choice Theory}

The subject chooses the position of an object in one of nine possible noisy squares (Figure 2); this is a 9-alternative, forced-choice (9-AFC) experiment. An automated computer algorithm estimates a detectability parameter $u$ using maximum likelihood and adapts contrast or dose so as to maintain an $80 \%$ probability of correct responses. In Appendix A, we describe details of how to obtain parameter estimates and uncertainties. The following is a brief summary.

To quantify object detectability, we used a signal detection model that assumes a continuous decision variable internal to the observer with Gaussian probability density functions for the choices "object present" and "object not present." These two overlapping Gaussian distributions have means that are separated by a "distance," $d$ ', that is a measure of detectability. In our studies, X-ray dose $(D)$ and the contrast between object and background $(C)$ are variables, giving

$$
d^{\prime}=u C \sqrt{D}
$$

where $u$, a variable name following the convention of Ohara et al. (1989), is the detectability parameter to be estimated. For fixed object contrast and dose, it becomes easier to discriminate between object and no-object as $u$, and hence $d^{\prime}$, increases. In our experiments, the value of $u$ depends upon independent variables such as object size and pulsed acquisition rate.

Ohara et al. (1989) derived the probability of detection for an $M$-AFC experiment, and their result is reproduced

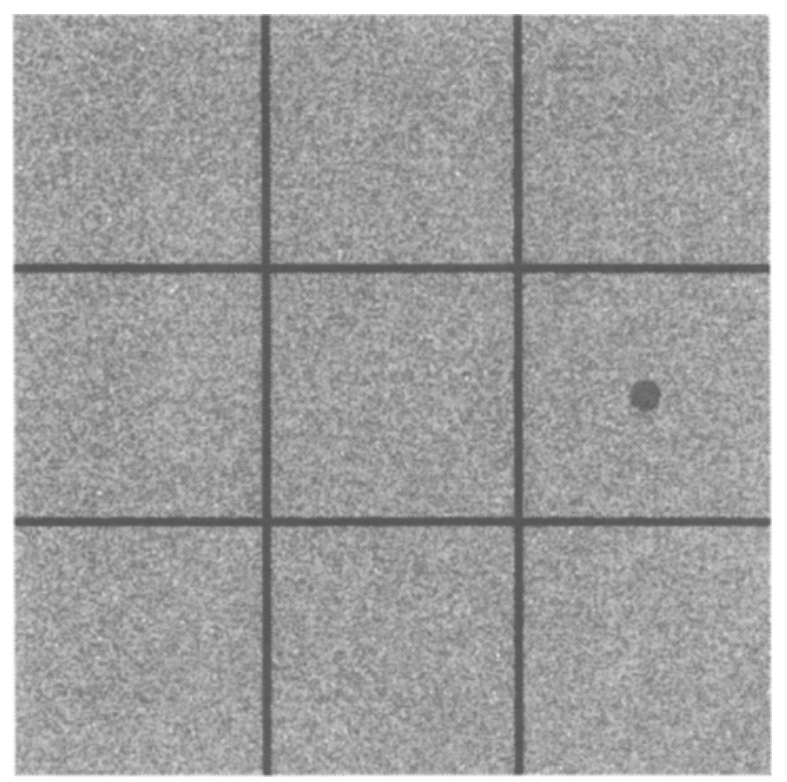

Figure 2. In this 9-alternative-forced-choice phantom, a disk of 16 pixels in diameter is randomly placed at the center of one of the squares. The noise in the image sequence obscures the disk, and the subject correctly or incorrectly chooses the square containing the disk. Noise or contrast levels are adapted as described in the text. For clarity, the disk contrast is set high in this figure. in Equation A2. This leads to the log likelihood function given in Equation A5. When this nonlinear function is maximized, we obtain the optimal, maximum-likelihood estimate of $u$, the measure of detectability.

For the nonadaptive case, Burgess (1995) investigated the variance of detectability estimates. In Appendix A, we extend this analysis to adaptive experiments and determine a method to calculate the coefficient of variation of $u, \sigma_{u} / u$ (Equation A11). Similarly, we develop coefficients of variation for other parameters of interest, namely contrast, $\sigma_{C} / C$ (Equation A12) and dose, $\sigma_{D} / D$ (Equation A14). Since methods are linked to find an EPD for test, $\sigma_{D} / D$ depends upon both reference and test presentation data.

\section{METHOD}

Computer programs for the reference/test adaptive method are described in Appendix B. Below, we describe Monte Carlo methods for testing the program and uncertainty calculations. This is followed by a description of human subject experimental methods.

\section{Monte Carlo Simulations}

To test the adaptive computer algorithms and to verify parameter uncertainties, Monte Carlo simulations were performed. The human subject was replaced with a computer "simulated subject" that produced realizations of the random decision variables internal to the observer. For each of the nine possible positions of the phantom, we assumed a Gaussian probability density function for the decision variable. In the position containing the object, the mean of the Gaussian was $d^{\prime}$; the mean at the other eight positions was zero. Recall that $d^{\prime}$ was a normalized distance in terms of standard deviations. Simulations mimicked human subject experiments. To perform a trial, the adaptive forced-choice code randomly picked a position for the object. The code simulating the subject then generated eight random decision variable values for the positions without the object and one for the position with the object. The highest value was rightly, or wrongly, chosen as the position of the object. The adaptive forced-choice code used this and previous measurements to obtain parameter estimates and adjust contrast or dose values for the next trial. This implementation correctly gives the psychometric curve in Equation A2. We simulated adaptive-dose, adaptive-contrast, or interspersed reference/test experiments. Typically, we ran 1,000 Monte Carlo repetitions of an experiment consisting of 100 trials. Parameter estimates were obtained as a function of trial number. Means and standard deviations over the 1,000 Monte Carlo repetitions were computed as a function of trial number.

\section{Display Workstation}

We used a new display system designed for perception experiments consisting of software running on a conventional PowerMac 7100 personal computer (Apple Computer, Inc., Cupertino, CA). A single image frame could be repeatedly displayed any integer number of display frame times. Repeatedly looping cine displays were made from image frames stored in RAM, and the quantity of RAM was the only limit on the number of new noisy image frames. For example, in our experiments consisting of 100 frames of $384 \times 384$ pixels, $30 \mathrm{MB}$ of RAM were required (approximately 14.1 $\mathrm{MB}$ for the cine loop images, $10 \mathrm{MB}$ for a large, precomputed noise array, and $6 \mathrm{MB}$ for the display program and system). The display was carefully linearized using a SuperMatch Calibrator System (SuperMac Technology, Sunnyvale, CA), and the maximum screen luminance was set at $18.9 \mathrm{ftL}$. In the current experiments, both reference and test were displayed at an accurate rate of 66 frames $/ \mathrm{sec}$ using the vertical synch pulse on the PowerMac as a time standard. 
Although reference actually mimicked $33 \mathrm{acq} / \mathrm{sec}$ and test mimicked $16.5 \mathrm{acq} / \mathrm{sec}$, we refer to them as the X-ray standards of pulsed-30 and pulsed-15, respectively.

\section{Phantom}

The 9 -AFC phantom was a $384 \times 384$ pixel image divided into nine squares, each having $128 \times 128$ pixels (Figure 2 ). A lowcontrast dark disk was placed randomly in one of the nine square centers. The contrast of the disk, $C$, was

$$
C=\frac{\left|\mu_{b}-\mu_{d}\right|}{\mu_{b}+\mu_{d}}
$$

where $\mu_{b}$ and $\mu_{d}$ were the mean gray-scale value of the background and disk, respectively. In an eight-bit display, the value of $\mu_{b}$ was 128 gray levels and $\mu_{d}$ varied from 113 to 127 gray levels. We assumed a Poisson distribution of X-ray quanta (Aufrichtig, Xue, et al., 1994). To mimic an $X$-ray system, dose was changed while maintaining the same mean gray level. To save time and disk space, we precomputed noise values corresponding to a dose $1.0 \mathrm{Q} / \mathrm{acq}$ and adjusted them for appropriate pixel gray level and dose. In the case of background pixels, a dose $1.0 \mathrm{Q} /$ acq corresponded to a standard deviation of 29.2 gray levels. At a dose $r Q /$ acq, background noise variance was $(29.2)^{2} / r$. Noise excursions were relatively large and average values gave desired real-value contrasts.

\section{Experiments}

A description of a single experiment follows. First, 50 reference $(<\mathrm{R}\rangle)$ presentations with a nominal dose $1.0 \mathrm{Q} /$ acq were given and contrast was adapted to get $80 \%$ probability correct. Second, 50 test $(<\mathrm{T}\rangle)$ presentations with contrast from the reference display were given and dose was adapted to get $80 \%$ probability correct. Third, reference and test were alternated $(<\mathrm{R} / \mathrm{T}\rangle)$ while adapting the reference contrast and the test dose, respectively. The final test dose was equal to EPD.

The disk diameter was 16 pixels with $0.4 \mathrm{~mm}$ /pixel; the viewing distance was $1 \mathrm{~m}$; and the disk occupied $0.32^{\circ}$ of visual angle. The number of frames in a continuous loop was 100 .

Subjects were instructed to take as long as needed, and the presentation was terminated on a mouse-press response. Subjects were urged to make the most accurate response possible, and they were given positive feedback with a computer beep when the response was correct. Except in preliminary experiments, subjects were aware that the response time was recorded. Experiments were conducted in a darkened room.

\section{RESULTS}

\section{Simulations}

Burgess (1995) reported that parameter uncertainty in a forced-choice experiment depends upon the probability of correct choice. We investigated this for the case of a nonadaptive, or fixed, forced-choice experiment. In Figure 3, we plot $\sigma_{u K} / u_{K}$ as a function of $p$ for $K=100$. (To make this calculation, $d_{K}^{\prime}$ was varied from 0 to 5 , and $p$ and $\sigma_{u K} / u_{K}$ were calculated from Equations A2, A8, and A11.) As compared with the $M=9$ curve, the $M=4$ curve was more narrow and displaced upward to a higher variance. For these reasons, we used $M=9$ in our adaptive experiments (see Discussion section). For the case of $M=9$, the minimum of $\sigma_{u K} / u_{K}$ was $6.92 \%$ at a probability correct of $87 \%$. We chose our adaptive operating point at $80 \%$, where the value of $\sigma_{u K} / u_{K}$ was $7.06 \%$.

We performed a Monte Carlo model-to-model simulation for an interspersed reference/test experiment. Ref-

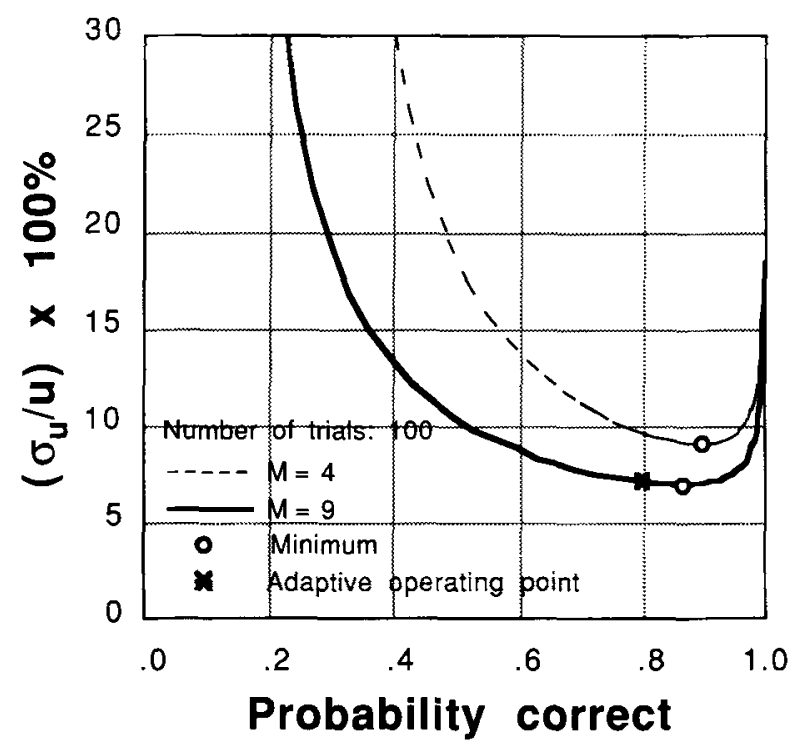

Figure 3. In a forced-choice experiment, the uncertainty of parameter estimates depends upon the probability of a correct choice. For the nonadaptive case (Equation A11), the coefficient of variation of $u, \sigma_{u} / u$ is plotted as a function of the probability correct, $p$, for $K=100$. For $M=9$, the curve has a relatively broad valley, and the minimum $(6.92 \%)$ occurs at $p=.87$. For $M=4$, the valley is narrowed and the minimum (8.89\%) occurs at $p=.90$. The adaptive operating point at $p=.80$ is near the minimum for $\boldsymbol{M}=9$.

erence was an adaptive-contrast experiment characterized by $u_{R}$, whereas test was an adaptive-dose experiment characterized by $u_{T}$. Simulation parameters were $u_{R}=100$ and $u_{T}=81.65$, giving EPD $=1.5$. Mean estimated values were plotted as a function of trial number (Figure 4). Final estimates were $u_{R}=(100.4 \pm 7.46), u_{T}=(82.1 \pm$ $6.03)$, and $\mathrm{EPD}=(1.51 \pm 0.32) \mathrm{Q} /$ acq. All parameter estimates were quite constant after 75 trials and were within $0.8 \%$ of true values, indicating no bias from the interspersed method. Interestingly, the average $C, u_{R}$, and EPD curves in the figure were all initially biased high, and this was a tendency in many of the Monte Carlo simulations. This occurred despite using uniformly distributed initial values. This probably arose from our clipping method (Appendix B) and because $u$ and $C$ values were always positive. There was no significant correlation of initial values with final estimates, absolute error, or estimated variance.

We investigated parameter uncertainties in detail and plotted various coefficients of variation as a function of trial number (Figure 5). Curves were relatively smooth, indicating that 1,000 Monte Carlo repetitions were probably sufficient for most analyses. All curves trended downward with increasing trial number, as expected for an uncertainty measure. The dependence was roughly and inversely proportional to the square root of total number of trials, as predicted theoretically for the nonadaptive case (Equation $\mathrm{A} 10$ ). Following the first 50 trials, the test and reference $u$ curves (Figures $5 \mathrm{a}$ and $5 \mathrm{~b}$, respectively) were very similar. Despite the linkage of 


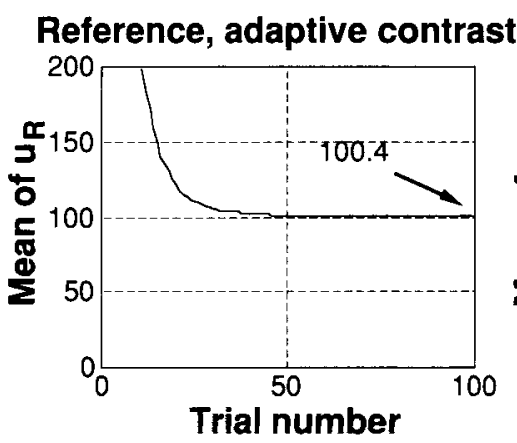

(a)

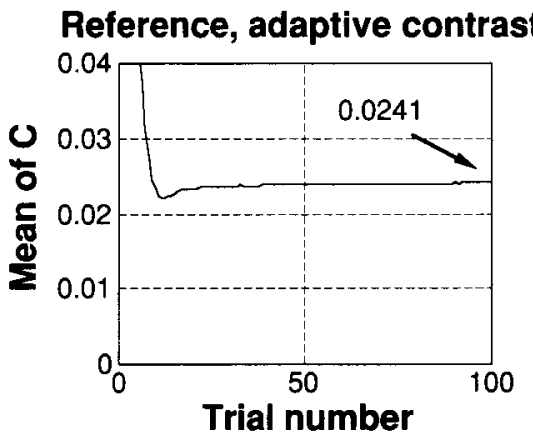

(c)

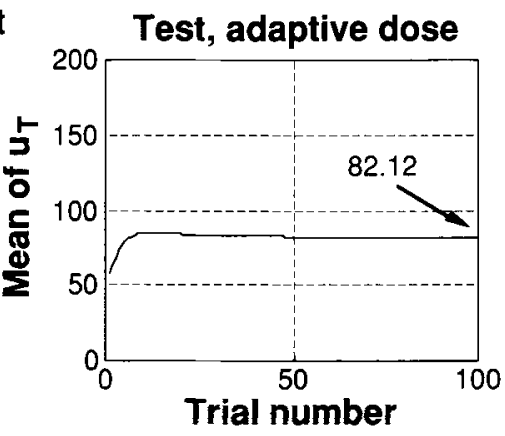

(b)

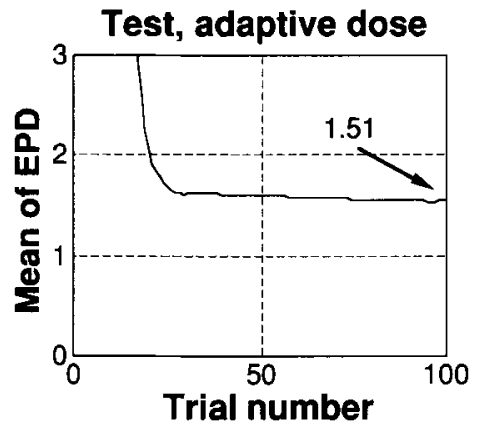

(d)

Figure 4. In a Monte Carlo, model-to-model test, we demonstrate the ability to estimate true parameter values. There are 1,000 repetitions of a Monte Carlo simulation; the panels show averages as a function of trial number: $u_{R}(\mathrm{a}), u_{T}(\mathrm{~b})$, contrast (c), and equivalent perception dose (EPD) (d). Final estimates (true values) are $u_{R}, 100.4$ (100.0); $u_{T}, 82.12$ (81.65); contrast, 0.0241 (0.02405); and EPD 1.51 (1.50) Q/acq.

the contrast variable, end values were almost identical. As theoretically predicted (Equation A12), the coefficient of variation of $C$ was similar to that for $u$. On the other hand, the coefficient of variation for EPD was greater, as predicted theoretically (Equation A14).

From the Monte Carlo data, we made various standard deviation calculations (Table 1). Our "gold standard" was final parameter uncertainties computed directly over the 1,000 Monte Carlo simulations at $K=100$ (second column, Monte Carlo). In the fourth and fifth columns (Single Repetition), parameter uncertainties were calculated from single experiments of $K$ trials using Equation A9. Over the 1,000 repetitions, we obtained $\sigma_{u R} / u_{R}=(7.30 \pm$ $0.22) \%$ (single repetition), which agreed favorably with the gold standard of $7.43 \%$ (Monte Carlo). Other column entries for single repetition also agreed favorably; for example, $\sigma_{\mathrm{EPD}} / \mathrm{EPD}=(20.62 \pm 0.42) \%$ (single repetition) agreed favorably with $21.12 \%$ (Monte Carlo). Interestingly, adaptive results were close to nonadaptive (fixed) theoretical predictions. For example, we found that $\sigma_{u T} / u_{T}=7.06 \%$ (fixed) agreed well with $7.34 \%$ (Monte Carlo). Obviously, adaptation only slightly increased parameter uncertainty. Because of the ease of computation, it was attractive to compute standard deviations from the last several estimates, as done in columns 6 and 7 (last 50). Comparing results, we found that $\sigma_{u R} / u_{R}=7.43 \%$ (Monte Carlo) was much larger than $(3.12 \pm 1.52) \%$ (last
50 ), and all other "last 50 " values greatly underestimated the true parameter uncertainty. We hypothesized that parameter estimates obtained as a function of trial number were correlated, and this led to the underestimation.

We were interested in the range of experimental standard deviations that might be obtained, and we studied this in simulations. In order to normalize for the number of trials performed, we created ratios from each repetition: $\sigma_{u R} /\left(\sigma_{u R}\right)_{\text {fixed }}, \sigma_{u T} /\left(\sigma_{u T}\right)_{\text {fixed }}$, and $\sigma_{\mathrm{EPD}} /\left(\sigma_{\mathrm{EPD}}\right)_{\text {fixed }}$. [Values for $\left(\sigma_{\mathrm{EPD}}\right)_{\text {fixed }}$ were obtained using fixed results for $\sigma_{u R}$ and $\sigma_{u T}$ in Equation A14.] In Figure 6, histograms were plotted, and $95 \%$ of values were below calculated thresholds of 1.11 for both $\sigma_{u R} /\left(\sigma_{u R}\right)_{\text {fixed }}$ and $\sigma_{u T} /\left(\sigma_{u T}\right)_{\text {fixed }}$ and 1.08 for $\sigma_{\mathrm{EPD}} /\left(\sigma_{\mathrm{EPD}}\right)_{\mathrm{fixed}}$.

\section{Human Subject Experiments}

In Figure 7, data from an adaptive reference/test experiment with 1 subject were plotted. Intervals $\langle R\rangle,\langle T\rangle$, and $<\mathrm{R} / \mathrm{T}>$ were identified, and the 100 trials on the $u_{R}$ curve consisted of the 50 initial reference trials plus the 50 trials alternated with test. Over the last 50 trials, the contrast for reference was adapted between 0.018 and 0.02 and linked to the contrast for the test display. The test dose adapted between roughly 1.3 and $1.5 \mathrm{Q} / \mathrm{acq}$. The final test dose gave EPD $=1.33 \pm 0.27 \mathrm{Q} / \mathrm{acq}$. Correcting for frame rate, this gave a per-minute dose saving of $33 \%$ for a pulsed-15 display. Response time data showed sig- 
Reference, adaptive contrast

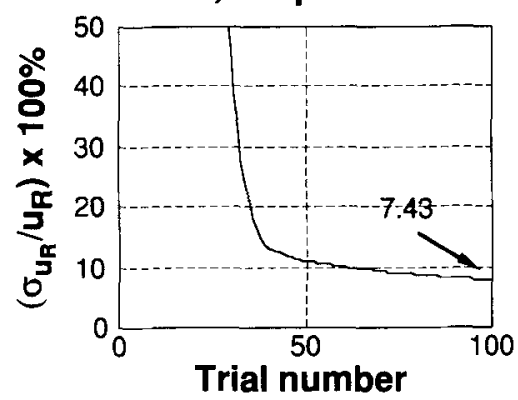

(a)

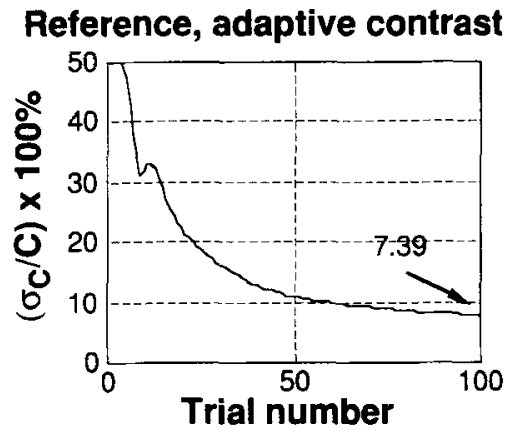

(c)

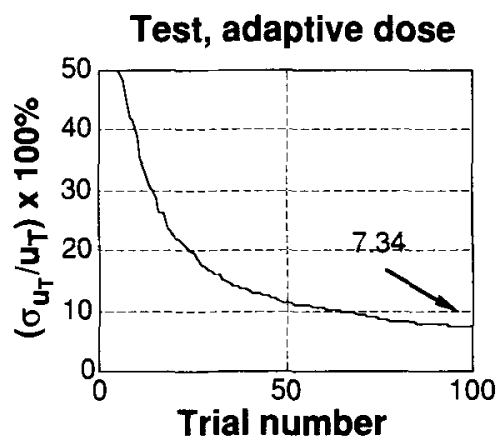

(b)

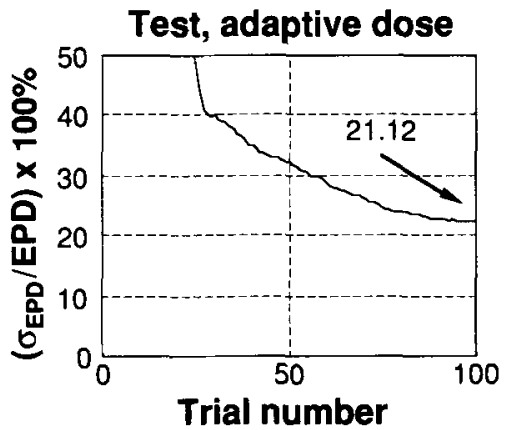

(d)

Figure 5. Coefficients of variation from Monte Carlo simulations are plotted as a function of trial number: $\sigma_{u R} / u_{R}(\mathrm{a}), \sigma_{u T} / u_{T}(\mathrm{~b}), \sigma_{C} / C$ (c), and $\sigma_{\mathrm{EPD}} / \mathrm{EPD}$ (d). Data (final values) are from the same 1,000 repetitions as those in Figure 4.

nificant overlap (Figures $7 \mathrm{e}$ and $7 \mathbf{f}$ ). Mean response times computed over the last 50 values $(11.5 \pm 5.4 \mathrm{sec}$ for reference and $14.0 \pm 5.7 \mathrm{sec}$ for test) were not statistically different (Student $t$ test at a .05 significance level).

In this interspersed experiment, subject attention and accuracy criteria should be the same for both reference and test presentations. There are many reasons to believe that this will allow improved comparisons of measurements. To quantitatively investigate the improvement and to compare measurement standard errors with theory, we repeated the reference/test experiment on several days. These data allow us to compare the variability of interspersed measurements with the variability from different days.

The reproducibility of a subject was investigated over five experiments on different days (Figure 8). Although all data were reasonably stable at the end of 100 trials, there was considerable variation from 1 day to the next. For example, with regard to $u_{R}$, the Day 1 estimate (181.4 \pm 13.2) was much greater than that on Day $2(149.5 \pm 10.5)$. Not only was there no overlap of final estimates, but there was virtually no overlap of uncertainties over the most of 100 trials. Rather than using such absolute values, we prefer to compare interspersed measurements using a ratio such as $\left(u_{R} / u_{T}\right)^{2}$. In the next paragraph, we quantitatively examine the advantage of comparing measurements from the same session.

Further analysis of the data from Figure 8 is shown in Figure 9. From final maximum-likelihood estimates on
5 days, means and standard deviations were $u_{R}=180.2 \pm$ $17.7, u_{T}=143.2 \pm 6.4$, and EPD $=(1.59 \pm 0.21) \mathrm{Q} / \mathrm{acq}$ (Figures 9a, 9b, and 9c). Coefficients of variation were $9.8 \%, 4.5 \%$, and $13 \%$, respectively, and values for $u_{T}$ and EPD were less than predicted by theory and simulation (Table 1). We also investigated the range of EPD values obtained by all other possible pairings of $u_{T}$ and $u_{R}$ measurements from different days. Using Equation 1 for both adaptive contrast and adaptive dose with the same contrast and $d_{80 \%}^{\prime}$, one obtains EPD $=\left(u_{R} / u_{T}\right)^{2}$. Over

Table 1

Various Coefficients of Variation From Simulated Data

\begin{tabular}{|c|c|c|c|c|c|c|}
\hline \multirow[b]{2}{*}{ Variable } & \multirow{2}{*}{$\begin{array}{l}\text { Monte } \\
\text { Carlo } \\
100 \%\end{array}$} & \multirow{2}{*}{$\begin{array}{l}\text { Fixed } \\
100 \%\end{array}$} & \multicolumn{2}{|c|}{$\begin{array}{c}\text { Single } \\
\text { Repetition }\end{array}$} & \multicolumn{2}{|c|}{ Last 50} \\
\hline & & & $100 \%$ & $S D$ & $100 \%$ & $S D$ \\
\hline$\sigma_{u R} / u_{R}$ & 7.43 & 7.06 & 7.30 & 0.22 & 3.12 & 1.52 \\
\hline$\sigma_{C} / C$ & 7.39 & & 7.30 & 0.22 & 3.10 & 1.31 \\
\hline$\sigma_{u T} / u_{T}$ & 7.34 & 7.06 & 7.27 & 0.31 & 3.10 & 1.39 \\
\hline$\sigma_{\mathrm{EPD}} / \mathrm{EPD}$ & 21.12 & & 20.62 & 0.42 & 9.01 & 4.43 \\
\hline
\end{tabular}

Note-In the second column (Monte Carlo), coefficients of variation and means are computed directly from the 1,000 Monte Carlo repetitions. In the third column (Fixed), values for a nonadaptive, fixed experiment are computed (Equation A 11 ). In the fourth and fifth columns (Single Repetition), coefficients of variation are computed from each Monte Carlo repetition using the theory developed in Appendix A. Because this is computed for each repetition, a coefficient of variation over the 1,000 repetitions is calculated. In the sixth and seventh columns (last 50), values are computed over the last 50 estimates for each repetition. Once again, coefficients of variation are computed over the 1,000 repetitions. 


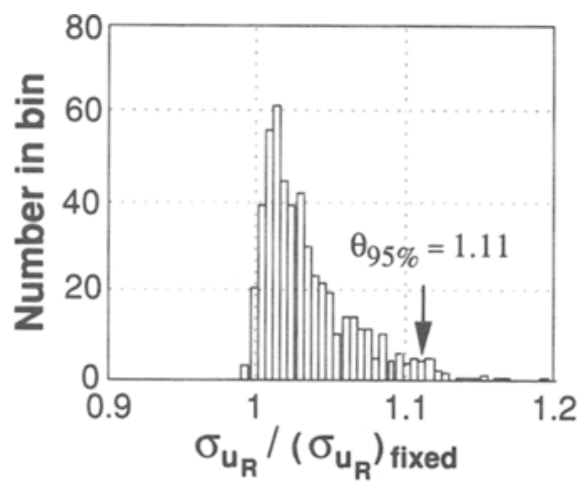

(a)

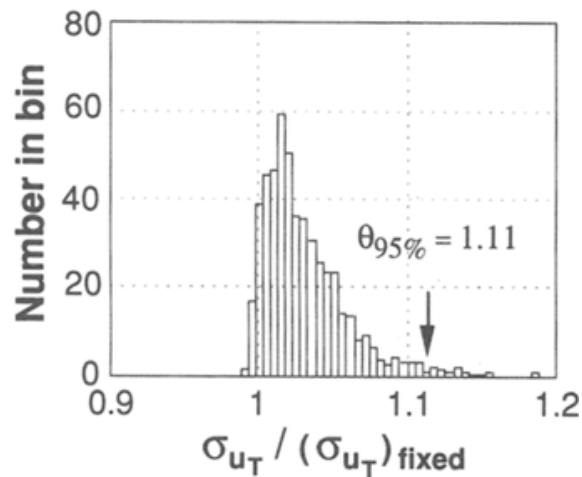

(b)

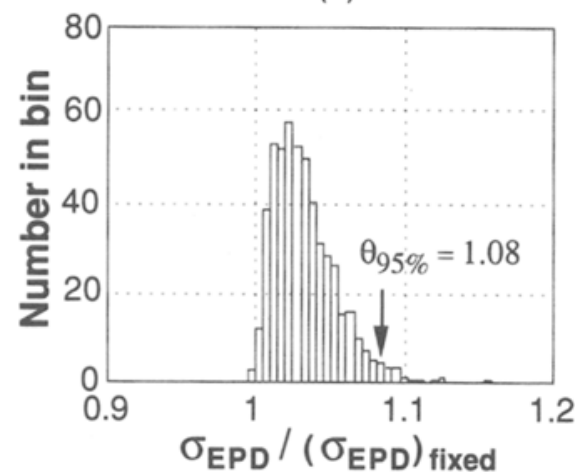

(c)

Figure 6. For each Monte Carlo repetition, a single repetition standard deviation is computed and normalized by the theoretical expectation for a fixed presentation of 100 trials: $\sigma_{u R} /\left(\sigma_{u R}\right)_{\text {fixed }}$ (a), $\sigma_{u T} /\left(\sigma_{u T}\right)_{\text {rixed }}(b)$, and $\sigma_{\text {EPD }} /\left(\sigma_{\text {EPD }}\right)_{\text {fixed }}(c)$. These are histograms and the vertical axis is the number in a bin. The bin size is 0.005 and the total number of repetitions is 1,000 .

the 20 new pairings, EPD values ranged from less than 1 to greater than $2 \mathrm{Q} / \mathrm{acq}$, and we computed $\mathrm{EPD}=1.60 \pm$ $0.33 \mathrm{Q} / \mathrm{acq}$ (Figure 9d). The standard deviation from pairings on different days $(0.33 \mathrm{Q} / \mathrm{acq})$ was approximately $50 \%$ greater than that from the interspersed data $(0.21 \mathrm{Q} / \mathrm{acq})$. However, for these limited number of data, an $F$ test did not show a significant difference between the standard deviations (.05 significance level).

Subject B was the most experienced subject. When the above analysis was done on a subject with less training (Subject $\mathrm{C}$ ), the standard deviation from pairings on dif- ferent days ( $1.14 \mathrm{Q} / \mathrm{acq})$ was double that for the interspersed reference/test pairings $(0.52 \mathrm{Q} / \mathrm{acq})$. The minimum to maximum range was three times.

We were also interested in differences in response times between reference (pulsed-30) and test (pulsed-15). Once again, we evaluated the interspersed methodology by comparing day-to-day variations with those from paired reference/test presentations. Response times were averaged over the last 50 trials for reference and test on 5 different days and plotted in Figure 10a. In all cases, bars for test were sliPerception \& Psychophysicsghtly higher than those for reference, indicating that the subject took slightly longer to respond for test. Even for this most experienced subject, there was significant variation of response times from 1 day to the next. To effectively reduce the effect of this variation, we computed ratios of response times for test over reference (Figure 10b). In this figure, all bars were above 1 . Combining data, the average was $1.17 \pm 0.10$, a value which is almost $2 S D$ s above 1.0 , the value for no effect. When we created the 20 other pairings from data on different days, the variation in the ratio was significantly increased (Figure 10c), and the average was $1.21 \pm 0.35$. Although the average was close to the previous value in Figure 10b, the standard deviation was increased 3.5 times. An $F$ test indicated a significant difference between the variances in Figures $10 \mathrm{~b}$ and $10 \mathrm{c}$ (.025 significance level). In the case of the reference/test pairs of response times, we found an increase with test (pulsed-15) that was virtually obscured in the day-to-day variations.

To facilitate comparisons with simulations, experimental results from Subject B are listed in Table 2. Data include EPD, detectability values (e.g., $u_{R}$ ), coefficients of variation of $u$ (e.g., $\sigma_{u R} / u_{R}$ ), and standard deviations normalized by the fixed standard deviation [e.g., $\sigma_{u R}$ / $\left.\left(u_{R}\right)_{\text {fixed }}\right]$. All experimentally determined standard deviations were calculated using the single repetition method. We were particularly interested in comparisons between experimental standard deviations and those from fixed in the 4th, 7th, and 10th columns. Values were all close to 1 , and the largest value was 1.04 . Standard deviation ratios for EPD in column 10 were all remarkably close to 1.0. All results agreed favorably with the simulated distributions in Figure 6.

\section{DISCUSSION}

There are several advantages of the adaptive reference/ test technique. First, interspersing reference and test presentations reduces the influence of extraneous experimental variables such as subject fatigue, attention level, and possible day-to- day physiologic variability. We, as well as others (Hall, 1981; Madigan \& Williams, 1987), think there is sometimes variation over the course of a single experiment. By alternating presentations within an interval of seconds, we reduced the uncertainty of EPD (Figure 9) and ratio of response times (Figure 10) and obtained more reliable experimental results. Second, by making experiments adaptive, we saved considerable time 
Reference, adaptive contrast

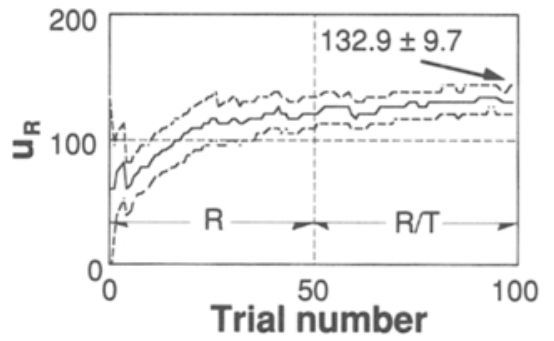

(a)

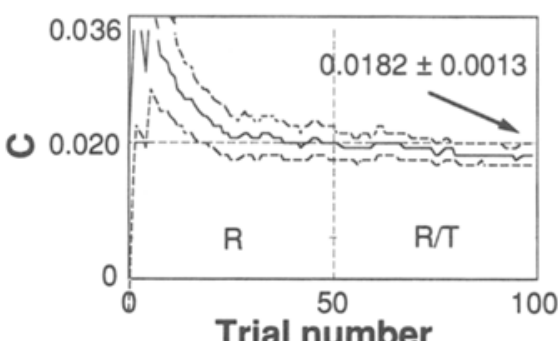

(c)

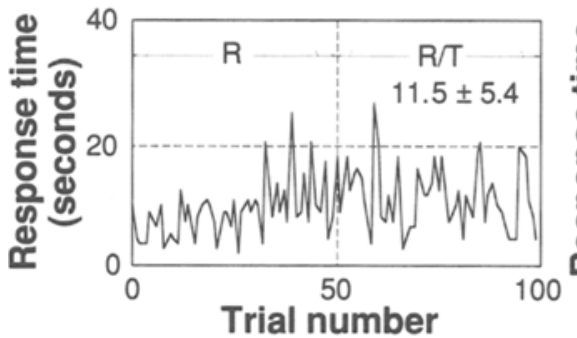

(e)

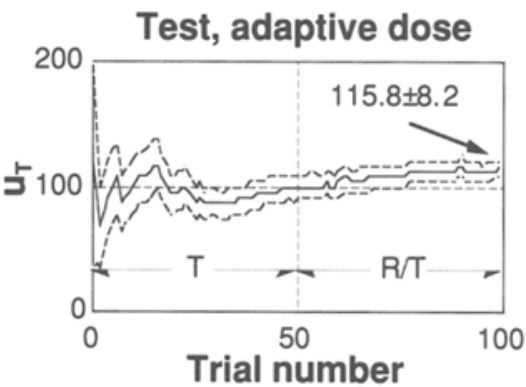

(b)

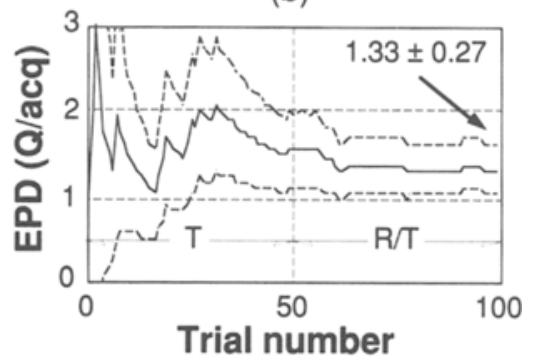

(d)

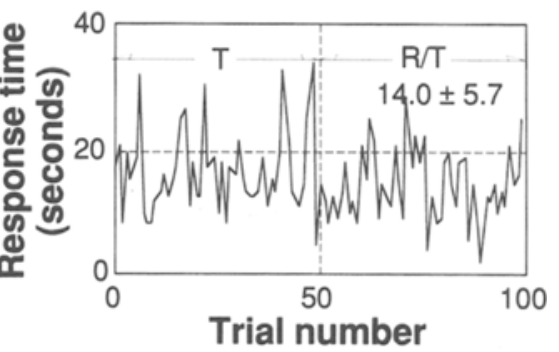

(f)

Figure 7. Data are from an adaptive reference/test experiment, and intervals $<R>$, $<\mathrm{T}\rangle$, and $<R / T>$ are identified as defined in the Method section. Trial numbers reflect the number of trials for the respective display; that is, the 100 trials on the $u_{R}$ curve consist of the 50 initial reference trials plus the 50 trials that were alternated with test. Curves are $u_{R}$ (a), $u_{T}$ (b), contrast of both reference and test (c), and equivalent perception dose (EPD) (d). Response times for reference and test are shown in (e) and (f), respectively. Final estimates and standard deviations are shown; for example, $\mathrm{EPD}=(1.33 \pm 0.27) \mathrm{Q} / \mathrm{acq}$. Response time averages were computed over the last 50 data points. Subject $A$.

trying to optimize experimental parameters for multiple subjects, instead molding the experiment to individual differences. Third, because we operated at a fixed probability correct for both reference and test, we eliminated a potential experimental variable. That is, EPD may depend upon the noise level and the probability correct, and a model with internal noise (Aufrichtig, Thomas, et al., 1994) demonstrates such a dependence. Fourth, because the probability correct is set, one can avoid a fixed forced-choice experiment at a low or a high probability correct. At a low or high probability correct, subjects can become bored, and one mistaken result due to inattention can greatly affect a detectability estimate (Burgess, 1995). Fifth, we eliminated the need to determine EPD graphically using an interpolation model (Aufrichtig, Thomas, et al., 1994; Wilson, Xue, \& Aufrichtig, 1994a, 1994b).

Table 2

Measurements From Subject B

\begin{tabular}{cccccccccc}
\hline Day & $u_{R}$ & $\sigma_{u R} / u_{R}$ & $\sigma_{u R} /\left(\sigma_{u R}\right)_{\text {fixed }}$ & $u_{T}$ & $\sigma_{u T} / u_{T}$ & $\sigma_{u T} /\left(\sigma_{u T}\right)_{\text {fixed }}$ & EPD & $\sigma_{\text {EPD }} /$ EPD & $\sigma_{\text {EPD }} /\left(\sigma_{\text {EPD }}\right)_{\text {fixed }}$ \\
\hline 1 & 181.41 & $7.27 \%$ & 1.03 & 144.49 & $7.12 \%$ & 1.01 & 1.58 & $20.35 \%$ & 1.02 \\
2 & 149.51 & $7.05 \%$ & 1.00 & 133.55 & $7.01 \%$ & 0.99 & 1.27 & $19.87 \%$ & 1.00 \\
3 & 188.87 & $7.05 \%$ & 1.00 & 151.03 & $7.16 \%$ & 1.01 & 1.57 & $20.10 \%$ & 1.01 \\
4 & 193.42 & $7.35 \%$ & 1.04 & 145.27 & $7.08 \%$ & 1.00 & 1.78 & $20.42 \%$ & 1.02 \\
5 & 187.68 & $7.09 \%$ & 1.00 & 141.75 & $7.02 \%$ & 0.99 & 1.77 & $19.95 \%$ & 1.00 \\
\hline
\end{tabular}

Note-Of particular interest are the ratios of experimental standard deviations to those from fixed-that is, $\sigma_{u R} /\left(\sigma_{u R}\right)_{\text {fixed }}$, $\sigma_{u T} /\left(\sigma_{u T}\right)_{\text {fixed }}$, and $\sigma_{\mathrm{EPD}} /\left(\sigma_{\mathrm{EPD}}\right)_{\text {fixed }}$. Values are all close to 1 , and the largest value is 1.04 . 

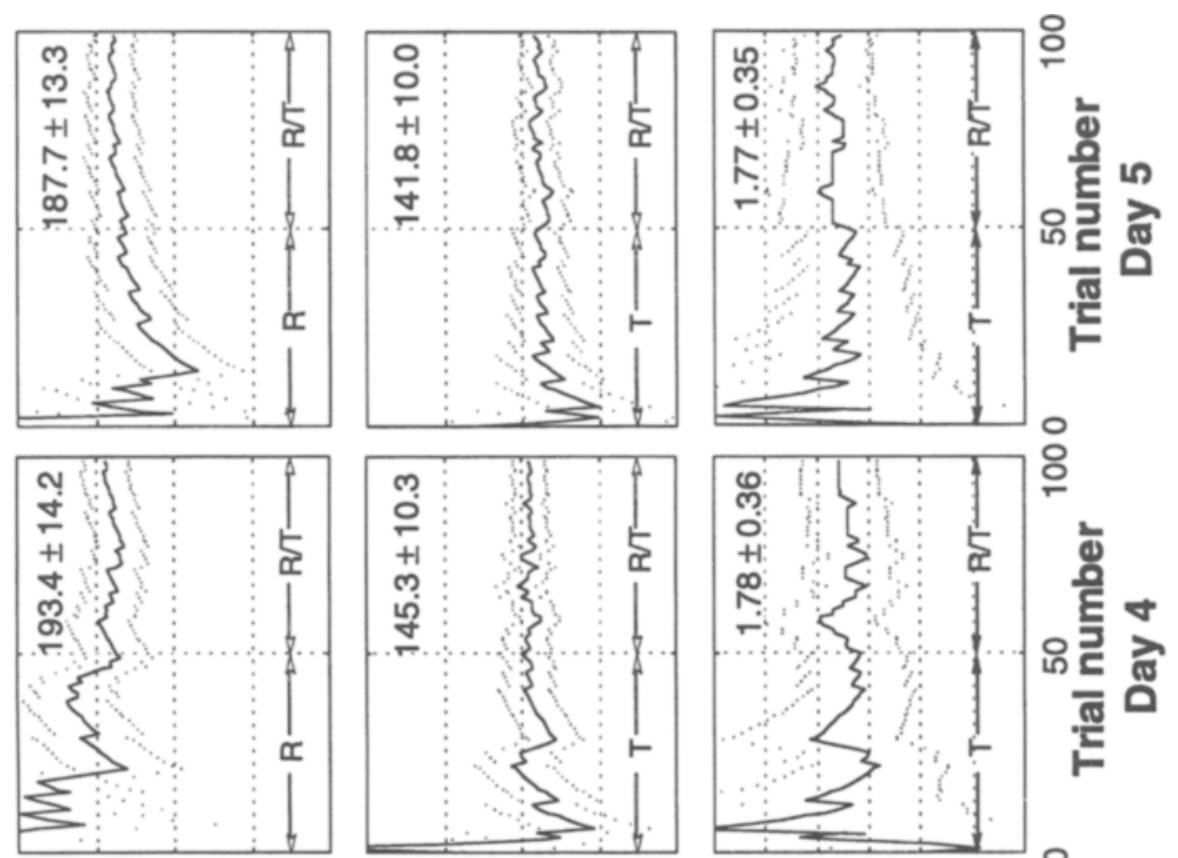

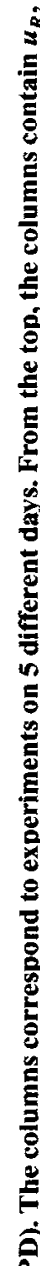
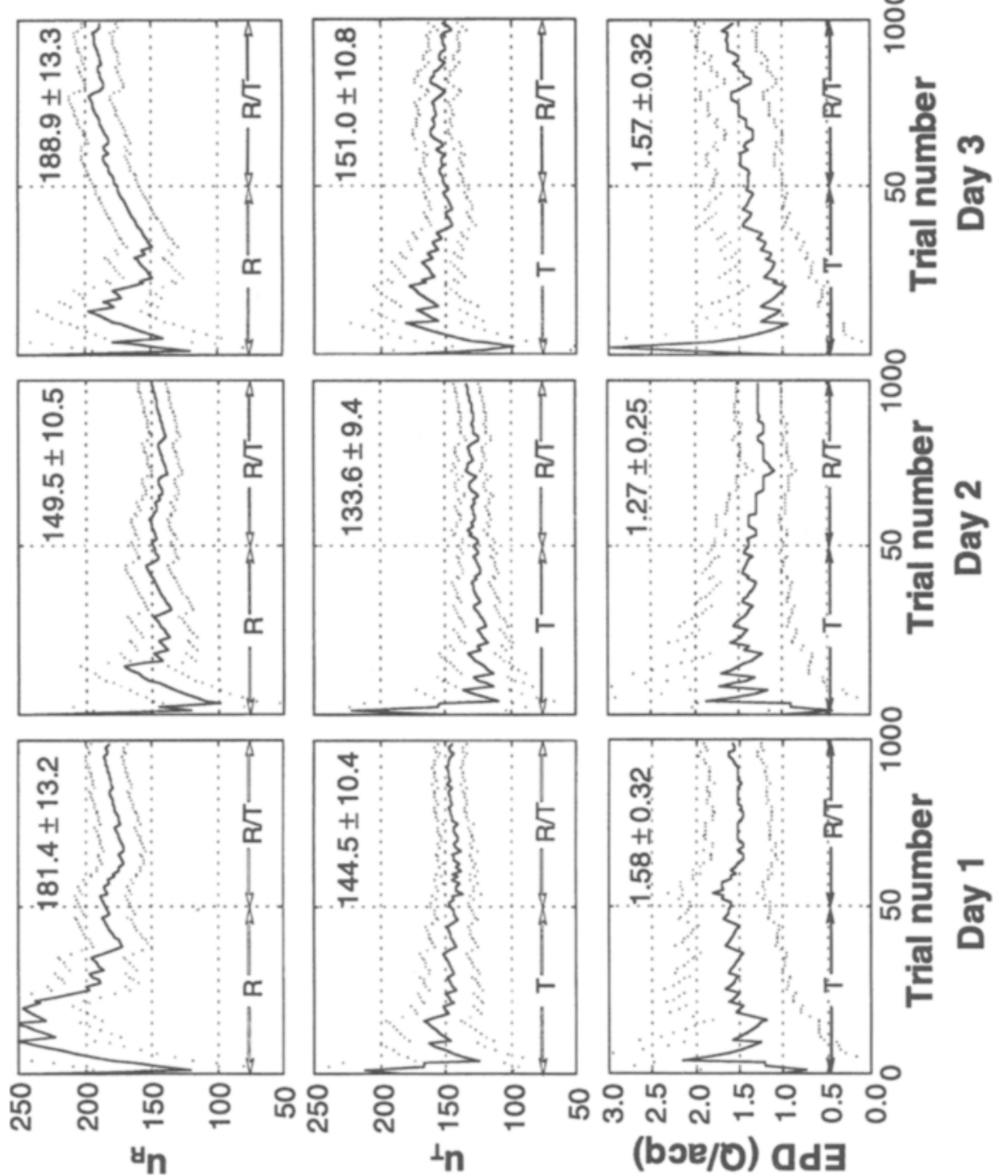

응
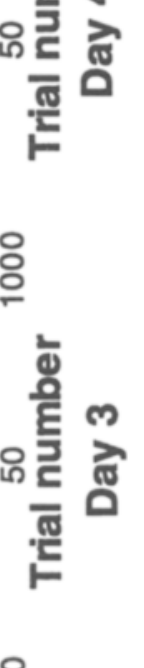


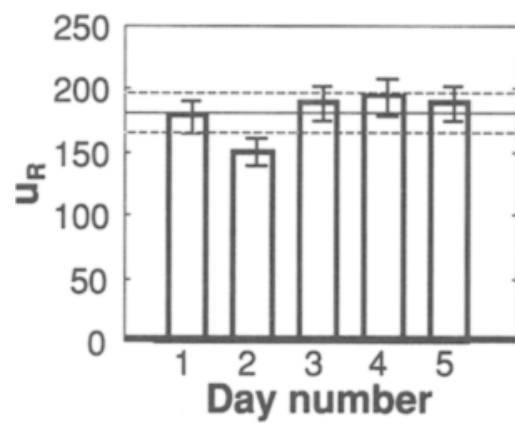

(a)

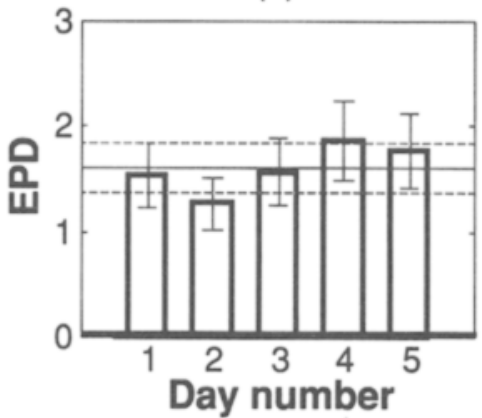

(c)

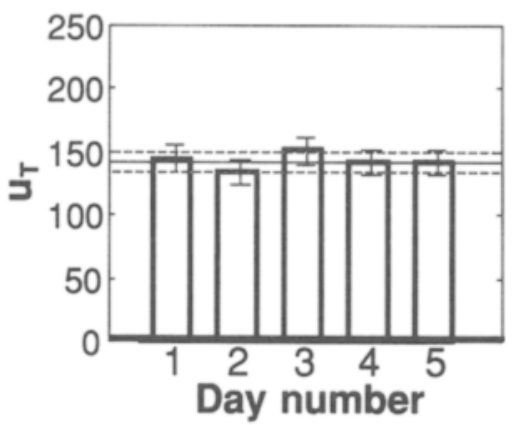

(b)

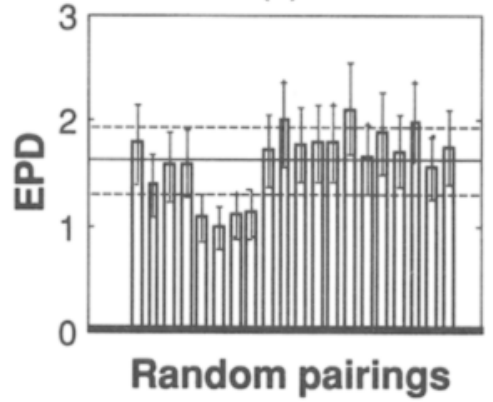

(d)

Figure 9. Further analysis of estimates from Figure 8. The $u_{R}, u_{T}$, and equivalent perception dose (EPD) estimates are plotted for all 5 days in (a), (b), and (c), respectively. In (d), all of the other 20 possible pairings of data on different days are used to compute EPD values. In each panel, means and standard deviations are plotted as solid and dashed horizontal lines, respectively. Subject B.

An interpolation model may be incorrect, and graphical analyses complicate the determination of parameter uncertainties. Sixth, the $M$-AFC method eliminates the subjectivity present in other paradigms and measures true detectability. Seventh, a comparison measurement should also reduce the effect of any bias toward certain positions in the display, a concern in conventional experiments (Blackwell, 1963; Swensson \& Judy, 1996).

There is an advantage to running reference and test experiments concurrently with a linked contrast experimental variable. Our goal was to obtain the equivalent perception dose, and this required that we perform measurements at the same object contrast. Obviously, we could have performed reference first and then run test at the same contrast. However, since reference is adaptive, it has a history of changing contrast values. It is preferable to use this same sequence of contrast values when making the test measurement, as in our concurrent measurement. A similar argument exists with regard to response times.

All of these attributes combine to give the single most useful aspect of the method. We can pose a question, run a single 1-h experiment on a single subject, and answer the question with considerable confidence.

The method applies to many of our experiments in Xray fluoroscopy. For example, we use the reference/test method to evaluate new acquisition (Xue \& Wilson, 1996) and image processing (Wilson, Jabri, Xue, \& Aufrichtig, 1996) methods. There are numerous similar applications in medical imaging (Wagner \& Brown, 1985) and image compression (Hearty, 1993). Many other applications exist in perception. For example, it is desirable to test contrast sensitivity with sine wave presentations and compare results with those from a more complex test like detection and recognition of letters of the alphabet (Gilmore, Thomas, Klitz, Persanyi, \& Tomsak, 1996). Using our method, one can present sine waves and adaptively vary contrast to obtain a threshold at a constant proportion correct, typically $80 \%$. One can also present letters, adaptively vary the contrast, and obtain a contrast threshold. By alternating presentations, one can ensure that the attention level of the subject is comparable in both tasks. The two display types can be presented independently of each other, or the most recent contrast from the contrast sensitivity test can be used to adjust contrast for the letter presentation. With this linking of variables, experiment time can be saved, a particularly important issue in a clinical test with patients.

\section{Detectability Estimates}

Many relate $d^{\prime}$ experimental values to models of signalto-noise-ratio (SNR) that depend upon experimental parameters such as contrast, object size, and background (Aufrichtig, Thomas, et al., 1994; Burgess, 1994, 1995; Burgess, Wagner, Jennings, \& Barlow, 1981; Rolland \& Barrett, 1992). With some independent variables fixed, many, but not all, models give the general form of Equa- 


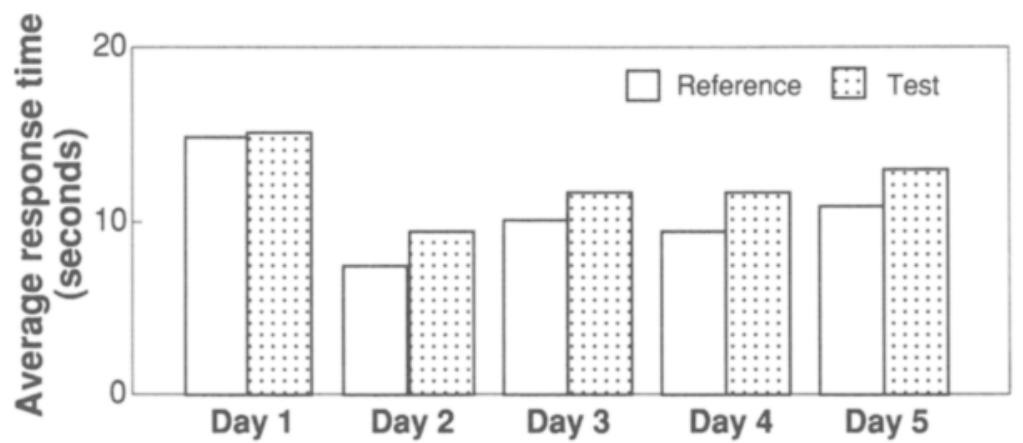

(a)

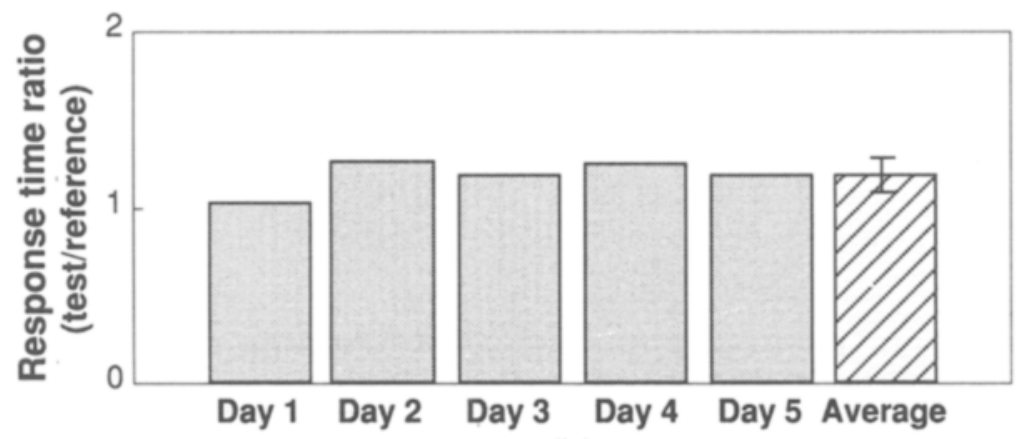

(b)

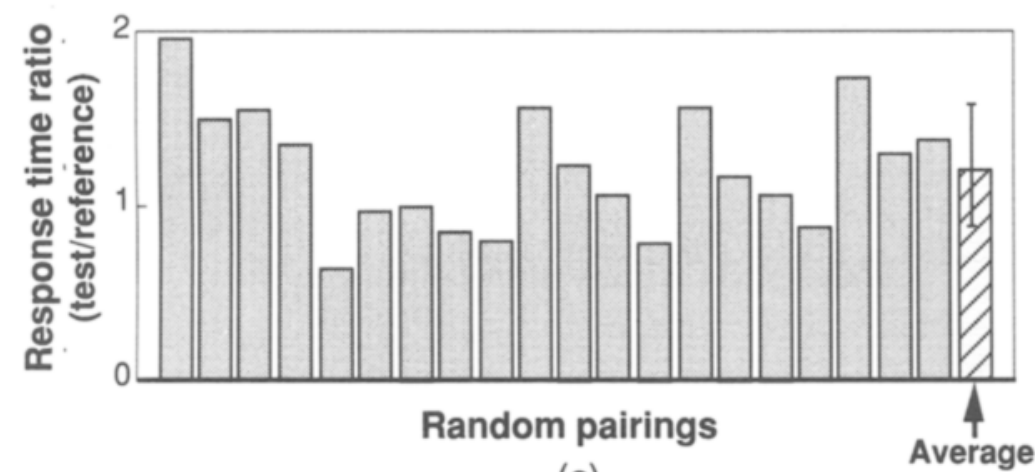

(c)

Figure 10. Response times measured from the data in Figure 8. Reference and test measurements are averages over the last 50 measurements and are plotted in Figure 10a. Figure 10b shows the ratio of test over reference. The bar labeled "average" gives the average ratio and standard deviation. Figure $10 \mathrm{c}$ shows 20 other response time ratios obtained by pairing data on different days. Comparison of average bars in Figures $10 \mathrm{c}$ and $10 \mathrm{~b}$ reveal a large day-to-day variation that is reduced by interspersed presentations. Subject $B$.

tion 1 (see Appendix A). However, the method does not depend upon the accuracy of Equation 1. Equation 1 is used to compute the next experimental value in the adaptive method. As such, it needs to be applicable only over a local operating range. In the case of EPD measurements, no model assumption is required. In other cases, it is desirable to obtain absolute detectability, $d^{\prime}$, as a function of independent experimental variables in order to compare to theories of SNR (Burgess, 1995). For example, it is very popular to measure absolute detectability as a function of contrast. Running multiple adaptive experiments at constant dose, or noise, one can obtain $d^{\prime}$ as a function of con- trast. Measurement variance can be shown on the contrast variable or propagated to the $d^{\prime}$ output.

In the case of an adaptive method, contrast or dose is varied, and the method does not operate at the exact minimum of the variance curve (Figure 3). Although an increased uncertainty is anticipated, Monte Carlo data in Table 1 and human subject data in Table 2 show that adaptation only slightly increases parameter uncertainty as compared with a nonadaptive (fixed) experiment at an optimal value.

As described by Maloney (1990), confidence intervals do not always follow theoretical distributions when one 
is using small numbers of trials and adaptive experiments, and a solution is to use bootstrapping techniques. Because of the computationally demanding psychometric function, bootstrapping is currently impractical for analysis of our experiments. The methods described in Appendix A and labeled (Single Repetition in Table 1) are more practical and appear to be quite adequate.

We are interested in using experimentally measured variances to improve the technique. One idea is to apply a variance threshold to reject experiments due to subject variability. Another is to use the variance measure as a stopping criterion. A third is to do outlier rejection to refine estimates. From simulations, the distribution of standard deviation ratios is very tightly distributed near 1.0 , and one can easily calculate $95 \%$ thresholds (Figure 6). Experimentally measured standard deviations tend to agree with simulated values (Table 2).

Using Monte Carlo methods, some compute efficiency for adaptive methods. Efficiency is effectively the inverse ratio of the adaptively measured parameter variance at a target probability to that obtained from idealized experiments fixed at a target probability. Taylor (1967) reported the efficiency of a PEST method to be $40 \%-50 \%$. Hall (1981) reported an efficiency of $77 \%$ for a 4 -AFC condition over 200 trials using a hybrid method. Watson and Pelli (1983) reported efficiency of a QUEST method as high as $84 \%$ after 128 trials for a $2-A F C$ condition. From data in Table 1, we computed efficiencies as the ratio of the fixed variance to the Monte Carlo variance. For 100 trials of a 9-AFC experiment, we calculated $u$ efficiencies of $90 \%\left[=(7.06 / 7.43)^{2}\right]$ and $93 \%\left[=(7.06 / 7.34)^{2}\right]$ for reference and test, respectively. Although one should compare the same number of trials and an identical number of choices (e.g., a 9-AFC), we are pleased that these values agree favorably with previous reports. One potential reason for the slightly improved efficiency as compared with that of Watson and Pelli is that continuously variable rather than discretely valued presentations were used.

Others emphasizing fast estimation and fewer trials have been concerned about bias with adaptive methods (Emerson, 1986; King-Smith et al., 1994; Madigan \& Williams, 1987; Simpson, 1989). For example, Emerson (1986) showed that a Bayesian method had less bias than a maximum-likelihood method with a fixed initial value, but after 50 trials, both estimates were very close to true values. We clipped initial contrasts in order to eliminate initial wild-point presentations and used a minimum of 100 trials. In this case, final estimates in Monte Carlo model-to-model tests were very close to true values. In addition, there was no correlation of the final estimates with initial values.

\section{Response Times}

With regard to absolute response time measurements, several potential complications (Luce, 1986) are reduced in the case of our comparison experiments. Most importantly, in any perception task one can normally trade off accuracy for the time to complete the task. Some measure this and call it the "speed-accuracy tradeoff function"
(Luce, 1986). This measurement necessarily requires significant experiment time, so others try to hold constant the accuracy criterion through instructions, information feedback, and data selection. Luce (1986, p. 57) has suggested that "there are reasons to doubt the actual success of this practice." We use instructions and information feedback (a computer beep) and compare reference and test measurements presumably taken under the same accuracy criterion. Note that we urge subjects to be accurate, and the resulting average response times are in the ceiling region of the speed-accuracy tradeoff function, where a change in response time should not greatly affect accuracy (Luce, 1986; Xue \& Wilson, 1996). Hence, detectability measurements should be quite valid. However, in this region, a wide range of response times will probably give the same accuracy. Therefore, we do not examine absolute response times; instead, we examine relative differences between reference and test presentations. Luce also pointed out that motivational variables, of which attention is a prime example, complicate all of the traditional response time experiments. Some believe that attention affects the sensory mechanism as well as the decision mechanism (Kahneman, 1973). By running experiments concurrently, our response time comparisons are made at the same attention level.

In the Results section, we compared response time and EPD ratios from concurrent measurements with those from different days. The variance obtained with concurrent measurements was reduced, indicating that the reference/ test method successfully reduces the effect of some of the experimental complications discussed above. One might hypothesize an increased response time variance from an adaptive method that presents variable contrast or dose values to the subject. However, this cannot be a significant effect because contrast or dose changed very little during the last 50 presentations, when response times were measured (Figure 7).

As advocated by Luce (1986), if one is particularly interested in absolute response times, one should measure the speed-accuracy tradeoff function. Typically, the average response time is varied by giving the subject various deadlines, and the accuracy is measured. An adaptive method is unsuitable because contrast is varied to give the same probability correct. To measure the speed-accuracy tradeoff, one needs a constant difficulty of detection. Given the difficulties in measuring absolute response times, we recommend that the adaptive method only be used for comparison measurements of response times.

\section{Method and Potential Improvements}

Experiments with image sequences impose severe computational and storage needs. In our experiments, image sequences have been displayed from computer memory, and sequence length is limited only by the quantity of relatively inexpensive RAM. The computer is sufficiently fast to create new, randomized noisy frames between display trials. Continuously valued contrasts and dose values are used rather than precomputed stimuli at discrete levels. We use a relatively complex psychomet- 
ric function that requires numerical integration and has therefore not been used previously in adaptive methods. Stored look-up table values speed the computation sufficiently to compute parameter estimates on the fly. Other, simpler, psychometric functions such as the logistic function (Hall, 1981; Pentland, 1980; Simpson, 1989; Taylor, 1967) and the Weibull function (King-Smith et al., 1994; Watson \& Pelli, 1983) are even faster to compute. The threshold theory by Blackwell (1963) provides yet another alternative. As argued previously, because the adaptive method uses small perturbations of the stimulus, the choice of a psychometric function is probably unimportant. We performed a numerical Monte Carlo experiment to verify model independence of EPD. We used Equation 1, $d^{\prime}=u C D^{1 / 2}$, and EPD $=1.5$ to generate "observer" data for both reference and test, and other models to estimate parameters and drive adaptation. The general form of the latter models was $d^{\prime}=u\left(u C D^{1 / 2}\right)^{n}$ where $n$ controls the slope at the operating point and its value is between 1.0 and 2.0 (Pelli, 1985). Despite this modeling error, the program gave a correct final EPD value. There was, however, a very small effect of modeling error on the convergence rate.

Nine rather than fewer alternatives are used because parameter uncertainty is reduced (Figure 3). A disadvantage is that it takes longer for one to visually scan all possible locations. Considering the number of trials to achieve an uncertainty, and the time per trial, it is possible to optimize $M$ in an $M$-AFC for the minimum total experiment time (Burgess, 1995). In our experiments with image sequences, the time to prepare a presentation is sufficiently long that reducing the number of alternatives will not greatly reduce experiment time. Another advantage of a 9-AFC as compared to a 4-AFC is that the uncertainty curve is flatter (Figure 3 ). Since the adaptive method varies contrast, or dose, about an operating point, a flatter curve should ensure more optimal results.

There are some potential improvements. A Bayesian method may reduce the number of presentations (Watson \& Pelli, 1983), and this modification may be added after gaining more experience. As suggested by KingSmith et al. (1994), for adaptation, the mean of the probability density function may be better than the maximumlikelihood estimate. For untrained subjects and/or patients, one might add wild-point editing using robust maximumlikelihood estimation techniques. It is desirable to use an experiment termination rule based upon experimental data, and a confidence interval is a good candidate. Some purposely impose small deviations in the stimulus variable in order to vary the task for the subject (Watson \& Pelli, 1983). We want to ensure a constant, high-performance level. In addition to the computer beep feedback, one might give a target $u$ value to subjects.

\section{Preliminary Experimental Results}

Preliminary adaptive experiments confirm our previous reports of dose savings with pulsed fluoroscopy (Aufrichtig, Xue, et al., 1994). Although dose savings depend upon the subject, for the 1 experienced subject studied in- tensively here, we found a $20 \%$ dose savings for pulsed15. For a disk of similar size, average results reported previously were $23 \%$ (Aufrichtig, Xue, et al., 1994).

To date, we have measured dose savings from an EPD. However, clinical X-ray dose also depends upon the time taken for a procedure. Although this is a clinical practice issue, one can begin to study it using subject response times (Whiting et al., 1994). The interspersed reference/ test method allows one to compare reference and test response times because it reduces the effect of such factors as training and fatigue and matches contrast and probability correct. Currently, we are analyzing response times in more detail.

\section{REFERENCES}

Aufrichtig, R., Thomas, C. W., Xue, P., \& Wilson, D. L. (1994). A model for perception of pulsed fluoroscopy image sequences. Journal of the Optical Society of America A, 11, 3167-3176.

Aufrichtig, R., Xue, P., Thomas, C. W., Gilmore, G. C., \& Wilson, D. L. (1994). Perceptual comparison of pulsed and continuous fluoroscopy. Medical Physics, 21, 245-256.

Bevington, P. R. (1969). Data reduction and error analysis for the physical sciences. New York: McGraw-Hill.

BLACKWELL, H. R. (1963). Neural theories of simple visual discriminations. Journal of the Optical Society of America, 53, 129-160.

BURgEss, A. E. (1994). Statistically defined backgrounds: Performance of a modified nonprewhitening observer model. Journal of the Optical Society of America A, 11, 1237-3176.

BuRgESs, A. E. (1995). Comparison of receiver operating characteristic and forced choice observer performance measurement methods. Medical Physics, 22, 643-655.

Burgess, A. E., Wagner, R. F., Jennings, R. J., \& Barlow, H. B. (1981). Efficiency of human visual signal discrimination. Science, 214, 93-94.

ElliotT, P. (1964). Forced choice tables. In J. A. Swets (Ed.), Signal detection and recognition by human observers (Appendix 1). New York: Wiley.

EMERSON, P. L. (1986). Observations on maximum-likelihood and Bayesian methods of forced-choice sequential threshold estimation. Perception \& Psychophysics, 39, 151-153.

FINDLAY, J. M. (1978). Estimates on probability functions: A more virulent PEST. Perception \& Psychophysics, 23, 181-185.

Gilmore, G. C., Thomas, C. W., Klitz, T., Persanyi, M., \& Tomsak, R. (1996). Contrast enhancement eliminates letter identification speed deficit in Alzheimer's disease. Journal of Clinical Geropsychology, 2, 307-320.

GREEN, D. M., \& SWETs, J. A. (1966). Signal detection theory and psychophysics. New York: Wiley.

HALL, J. L. (1981). Hybrid adaptive procedure for estimation of psychometric functions. Journal of the Acoustical Society of America, 69, 1763-1769.

HEARTY, P. J. (1993). Achieving and confirming optimum image quality. In A. B. Watson (Ed.), Digital images and human vision (pp. 149-162). Cambridge, MA: MIT Press.

Kahneman, D. (1973). Attention and effort. Englewood Cliffs, NJ: Prentice-Hall.

KELLY, D. H. (1979). Motion and vision: II. Stabilized spatio-temporal threshold surface. Journal of the Optical Society of America, 69, 1340-1349.

KELLY, D. H. (1984). Retinal inhomogeneity. I. Spatiotemporal contrast sensitivity. Journal of the Optical Society of America A, 1, 107-113.

King-Smith, P. E., Grigsby, S. S., Vingrys, A. J., Benes, S. C., \& Supowit, A. (1994). Efficient and unbiased modifications of the QUEST threshold method: Theory, simulations, experimental evaluation and practical implementation. Vision Research, 34, 885-912.

LUCE, R. D. (1986). Response times. New York: Oxford University Press.

Madigan, R., \& Williams, D. (1987). Maximum-likelihood psycho- 
metric procedures in two-alternative forced-choice: Evaluation and recommendations. Perception \& Psychophysics, 42, 240-249.

MALONEY, L. T. (1990). Confidence intervals for the parameters of psychometric functions. Perception \& Psychophysics, 47, 127-134.

Ohara, K., DoI, K., Metz, C. E., \& Giger, M. L. (1989). Investigation of basic imaging properties in digital radiography. 13. Effect of simple structured noise on the detectability of simulated stenotic lesions. Medical Physics, 16, 14-21.

PELLI, D. G. (1985). Uncertainty explains many aspects of visual contrast detection and discrimination. Journal of the Optical Society of America A, 2, 1508-1531.

PentLand, A. (1980). Maximum likelihood estimation: The best PEST. Perception \& Psychophysics, 28, 377-379.

Press, W. H., Flannery, B. P., Teukolsky, S. A., \& Vetterling, W. T. (1988). Numerical recipes in C: The art of scientific computing. New York: Cambridge University Press.

Rolland, J. R., \& BARRETT, H. H. (1992). Effect of random background inhomogeneity on observer detection performance. Journal of the Opical Society of America A, 9, 649-658.

Rose, A. (1973). Vision: Human and electronic. New York: Plenum.

SIMPSON, W. A. (1989). The step method: A new adaptive psychophysical procedure. Perception \& Psychophysics, 45, 572-576.

SwensSon, R. G., \& JuDY, P. F. (1996). Measuring performance efficiency and consistency in visual discriminations with noisy images Journal of Experimental Psychology: Human Perception \& Performance, 22, 1393-1415.

TAYLOR, M. M. (1967). PEST: Efficient estimates on probability functions. Journal of the Acoustical Society of America, 41, 782-787.

TAYLOR, M. M. (1971). On the efficiency of psychophysical measurement. Journal of the Acoustical Society of America, 49, 505-508.

WAGNER, R. F., \& BRowN, D. G. (1985). Unified SNR analysis of medical imaging systems. Physics in Medicine \& Biology, 30, 489518.

Watson, A. B., \& Pelli, D. G. (1983). QUEST: A Bayesian adaptive psychometric method. Perception \& Psychophysics, 33, 113-120.

Whiting, J. S., Eckstein, M., \& Eigler, N. L. (1994). Observer performance in dynamically displayed noisy image sequences [abstract]. Annals of Biomedical Engineering, 22, 46.

Wilson, D. L., Xue, P., \& Aufrichtig, R. (1994a). Perception in x-ray fluoroscopy. In H. L. Kundel (Ed.), Proceedings of SPIE Medical Imaging 1994: Image Perception, 2166, 20-23.

Wilson, D. L., Xue, P., \& Aufrichtig, R. (1994b). Perception of fluoroscopy last-image-hold. Medical Physics, 21, 1875-1883.

Wilson, D. L., Jabri, K. N., Xue, P., \& Aufrichtig, R. (1996). Perceived noise versus display noise in temporally filtered image sequences. Journal of Electronic Imaging, 5, 490-495.

XUE, P., \& WILSON, D. L. (1996). Pulsed fluoroscopy detectability from interspersed adaptive forced choice measurements. Medical Physics, 23, 1833-1843.

\section{APPENDIX A Theory}

\section{Estimation of Detectability}

To analyze experiments, we use a popular signal detection model (Burgess, 1995; Elliott, 1964), and we closely follow the derivation given by Ohara et al. (1989). The model hypothesizes that visual sensory inputs are converted to a single, continuous decision variable internal to the observer, with Gaussian probability density functions for the cases "object present" and "no object present." The density functions are

$$
\frac{1}{\sqrt{2 \pi}} \exp \left(-\frac{t^{2}}{2}\right)
$$

(no object)

and

$$
\frac{1}{\sqrt{2 \pi}} \cdot \exp \left(-\frac{\left(d^{\prime}-t\right)^{2}}{2}\right)
$$

where $d^{\prime}$, a detectability index, is the distance between the means of the two overlapping distributions as normalized by the standard deviation. The index is a decision-variable signal-to-noise ratio (SNR), and $d^{\prime}$ is often compared to various theoretical formulations that relate independent variables such as object size, background, and contrast to observer SNR (Burgess, 1995). For our adaptive technique, we use an approximate model that needs to be applicable over a small operating range. We assume that $d^{\prime}$ is proportional to the image contrast-to-noise ratio, $C / \sigma_{n}$, where $C$ is the contrast of the object as compared with background and $\sigma_{n}$ is the standard deviation of the display noise. The Rose (1973) model for a disk and the ideal observer model for a given object both have this form (Wagner \& Brown, 1985). In an X-ray system that maintains a constant gray level, $\sigma_{n}^{2}$ is inversely related to the number of X-ray quanta and hence X-ray dose, $D$ (Aufrichtig, Xue, et al,, 1994), and we have

$$
d^{\prime}=u C \sqrt{D}
$$

where $u$ is a detectability parameter to be determined.

One can derive the probability of a correct choice, $p\left(d^{\prime}\right)$ in an $M$-alternative, forced choice $(M$-AFC) experiment (Equation A15, in of Ohara et al., 1989), and the result is

$$
p\left(d^{\prime}\right)=\int_{-\infty}^{\infty}[\Phi(t)]^{M-1} \frac{1}{\sqrt{2 \pi}} \exp \left(-\frac{\left(d^{\prime}-t\right)^{2}}{2}\right) d t,
$$

where $\Phi(t)$ is the cumulative Gaussian distribution.

Using notation similar to that found in Appendix B of Ohara et al. (1989), at each value of the continuous variable contrast, $C_{k}$, we assume that $n_{k}=1$ for a correct response or $n_{k}=0$ for an incorrect response, where $k=1, \ldots, K$ and $K$ is the number of trials. If each decision is independent, the probability of getting a value $n_{k}$ at the $k$ th trial with contrast $C_{k}$ and dose $D_{k}$ is

$$
\operatorname{prob}\left(\boldsymbol{n}_{k}=n_{k}\right)=p_{k}^{n_{k}}\left(1-p_{k}\right) 1^{-n_{k}},
$$

where $p k=p\left(d_{k}^{\prime}\right)$. This binomial distribution has only two values: $\operatorname{prob}\left(\boldsymbol{n}_{\boldsymbol{k}}=1\right)=p_{k}$ and $\operatorname{prob}\left(\boldsymbol{n}_{\boldsymbol{k}}=\mathbf{0}\right)=1-p_{k}$. Considering all previous $K$ trials, the probability of the data set given $u$ is obtained:

$$
\begin{aligned}
\operatorname{prob}(\text { data set } \mid u) & =\operatorname{prob}\left(\boldsymbol{n}_{1}=n_{1}, \ldots, \boldsymbol{n}_{\boldsymbol{k}}=n_{k}, \ldots, \boldsymbol{n}_{\boldsymbol{K}}=n_{K} \mid u\right) \\
& =\prod_{k=1}^{K} p_{k}^{n_{k}}\left(1-p_{k}\right)^{1-n_{k}} .
\end{aligned}
$$

Taking logarithms, we get a $\log$-likelihood function:

$$
\mathrm{LL}(u)=\sum_{k=1}^{K}\left[n_{k} \log \left(p_{k}\right)+\left(1-n_{k}\right) \log \left(1-p_{k}\right)\right] .
$$

An optimal $u$ is obtained by maximizing this nonlinear function using an iterative optimization algorithm.

\section{Parameter Uncertainties}

Burgess (1995) described a method for determining the uncertainty in an $M$-AFC experiment with a fixed stimulus, and this is now extended to an adaptive reference/test experiment. For a trial $k$, the response is binomially distributed (Equation A3), and the variance is given below:

$$
\sigma_{p k}^{2}=p_{k}\left(1-p_{k}\right) \text {. }
$$

Dropping higher order terms, we can use standard error propagation equations (Bevington, 1969; Taylor, 1971). Using Equations $A 1$ and $A 6$, we obtain the variance for a forced-choice trial: 


$$
\sigma_{u_{k}}^{2}=\sigma_{p_{k}}^{2}\left(\frac{d u_{k}}{d p_{k}}\right)^{2}=\frac{p_{k}\left(1-p_{k}\right)}{\left(\frac{d p_{k}}{d d_{k}^{\prime}}\right)^{2}\left(\frac{d d_{k}^{\prime}}{d u_{k}}\right)^{2}}=\frac{p_{k}\left(1-p_{k}\right)}{\left(\frac{d p_{k}}{d d_{k}^{\prime}}\right)^{2} C_{k}^{2} D_{k}}
$$

where the derivative is obtained from Equation $\mathrm{A} 2$ :

$$
\frac{d p_{k}}{d d_{k}^{\prime}}=\int_{-\infty}^{\infty}[\Phi(t)]^{M-1} \frac{\left(t-d_{k}^{\prime}\right)}{\sqrt{2 \pi}} \exp \left(-\frac{\left(t-d_{k}^{\prime}\right)^{2}}{2}\right) d t .
$$

For all $K$ adaptive trials, the variance of $u_{K}$ is given below:

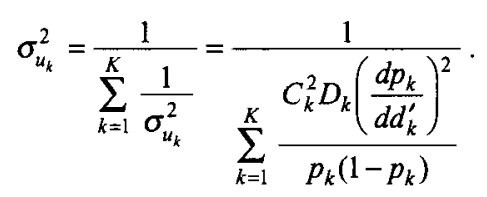

We obtain the maximum-likelihood estimate of $u_{K}$ using all previous responses at various $C$ and $D$ values and calculate $\sigma_{u_{K}}^{2}$ using Equations $\mathrm{A} 1, \mathrm{~A} 8$, and $\mathrm{A} 9$.

For the nonadaptive, fixed case, $C_{k}^{2}, D_{k}$, and $p_{k}$ are constants. We remove $k$ and the variance is simplified:

$$
\frac{\sigma_{u_{K}}^{2}}{u_{K}^{2}}=\frac{p(1-p)}{K d_{K}^{\prime}\left(\frac{d p}{d d^{\prime}}\right)^{2}}=\frac{\sigma_{d_{K}^{\prime}}^{2}}{d_{K}^{\prime 2}} .
$$

For this limiting case, using Equations $\mathrm{Al}$ and $\mathrm{A10}$, we obtain a coefficient of variation, $\sigma_{u_{K}} / u_{K}$, and find it equal to an expression for $\sigma_{d_{\kappa}^{\prime}} / d_{K}^{\prime}$, previously reported by Burgess (1995).

$$
\frac{\sigma_{u_{K}}^{2}}{u_{K}^{2}}=\frac{p(1-p)}{K d_{K}^{\prime}\left(\frac{d p}{d d^{\prime}}\right)^{2}}=\frac{\sigma_{d_{K}^{\prime}}^{2}}{d_{K}^{\prime 2}} .
$$

Several uncertainty relations are now developed for adaptive forced-choice procedures after $K$ trials. For the case of adaptive contrast and fixed dose, Equation Al can be rearranged as

$$
C_{K}=\frac{d_{80 \%}^{\prime}}{u_{c_{K}} \sqrt{D}}
$$

where $d_{80 \%}^{\prime}$ is a constant for an $80 \%$ probability of a correct response and $D$ is a constant. Assuming that adaptive changes of contrast do not affect the quality of $u$ estimates, the squared coefficient of variation of the contrast is obtained:

$$
\frac{\sigma_{C_{K}}^{2}}{C_{K}^{2}}=\frac{\sigma_{u_{C_{K}}}^{2}\left(\frac{d C_{K}}{d u_{C_{K}}}\right)^{2}}{C_{K}^{2}}=\frac{\sigma_{u_{C_{K}}}^{2}}{u_{C_{K}}^{2}} .
$$

In the case of an adaptive-dose, fixed-contrast experiment, a new dose value is computed from

$$
D_{K}=\frac{d_{80 \%}^{\prime 2}}{u_{D_{K}}^{2} C_{K}^{2}}
$$

and the squared coefficient of variation is given below:

$$
\frac{\sigma_{D_{K}}^{2}}{D_{K}^{2}}=\frac{\sigma_{u_{D_{K}}}^{2}\left(\frac{d D_{K}}{d u_{D_{K}}}\right)^{2}}{D_{K}^{2}}=\frac{4 \sigma_{u_{D_{K}}}^{2}}{u_{D_{K}}^{2}} .
$$

In the case of a linked EPD experiment, dose uncertainty is a function of $C_{K}$ and $u_{D_{K}}$ variances. Assuming independence of the two variances, standard error propagation techniques (Bevington, 1969) and Equation A12 are used to obtain the working equation:

$$
\frac{\sigma_{D_{K}}^{2}}{D_{K}^{2}}=\frac{\sigma_{u_{D_{K}}}^{2}}{D_{K}^{2}}\left(\frac{\partial D_{K}}{\partial u_{D_{K}}}\right)^{2}+\frac{\sigma_{C_{K}}^{2}}{D_{K}^{2}}\left(\frac{\partial D_{K}}{\partial C_{K}}\right)^{2}=4\left(\frac{\sigma_{u_{D_{K}}}^{2}}{u_{D_{K}}^{2}}+\frac{\sigma_{u_{C_{K}}}^{2}}{u_{C_{K}}^{2}}\right)
$$

The careful reader will notice that we estimate contrast and dose based on $u$ values from the previous trial. Hence, for a computer algorithm, we might modify equations after Equation A1 1 in such a way that contrast and dose are at step $(K+1)$ and $u$ is from step $K$, where $K$ is 100 in most experiments.

\section{APPENDIX B Computer Programs}

We now describe an adaptive-contrast, 9-AFC method, and then outline our changes for the adaptive-dose method. In the next section, we describe the linked reference/test technique.

\section{Adaptive Contrast Algorithm}

During the experiment, contrast is adjusted so as to maintain a constant $80 \%$ probability of correct detection. Using Equation $\mathrm{A} 2$, an $80 \%$ probability correct gives $d_{80 \%}^{\prime}=2.405$. The $80 \%$ value is purposely close to the minimum variance obtained at $87 \%$ (see Results section).

Figure B1 contains pseudocode describing the adaptivecontrast algorithm, and we use some of the variable and function names in the following description. In the main loop, noise data previously stored in files containing over 2 million floatingpoint noise values are loaded into a large 1-dimensional array, noise. As described later, these noise values are used to create image frames in the function create_image_sequence. Another function, adaptive_contrast, performs the adaptive process, and we typically initiate it with a constant, dose, an initial contrast, contrast, and a maximum number of trials, maxtrial. The entire image sequence is constructed in RAM and then displayed to the subject. At the end of every frame, the program checks for user inputs and terminates the display on a user mouse click.

Using the mouse on a graphical display, the subject identifies the position where he/she thinks the object is located. The program determines whether the response correctly identifies the position of the object and logs the result in Correct as true or false. If the response is correct, the program also beeps to reward the subject. (We find that the beep is one way to maintain subject attention.) The program stores results in an array, ResponseData, consisting of triplets of values (dose, contrast, correct) for each previous response. Maximum-likelihood estimates of detectability $u$ are obtained using a nonlinear optimization routine, Brent_optimization. The uncertainty $u$ Var is also calculated using $u$ and data recorded in ResponseData. Next, applying Equation 1 for an $80 \%$ probability correct, we calculate the contrast for the next trial. In the first few (approximately five) trials, contrast often varies significantly. To dampen fluctuations, the contrast for a new trial is limited to double or half the previous value. The loop is repeated using the new contrast value. In each loop, a new maximum-likelihood estimate of detectability $u$ is obtained using all previous responses. The experiment terminates when the maximum number of trials is reached, the operator terminates the experiment, or detectability variance 
uVar is less than a preset value. To date, we have used the first stopping criterion, and experiments have contained 100-200 image sequence presentations. Using precomputed noise values, the routine create_image_sequence quickly creates new image sequences. Zero-mean noise data are contained in an array, noise. Each noise value is scaled to mimic the desired $X$-ray dose and summed with another constant to create the desired brightness. Noise values are created and stored in BackgroundNoise and ObjectNoise. Note that in the case of adaptive contrast, background noise values are created only once in an experiment since dose, and hence the noise level, does not change. We carefully ensure randomization. For instance, to create image background, a value RandomStart is obtained from a random number generator, and a pixel row of data starting at RandomStart is block copied to the output frame. In the case of the object, noise values are copied on a pixel-by-pixel basis using RandomPixel as a pointer into the array ObjectNoise. The entire image sequence is created before exiting the routine.

We use Brent's iterative method (Press, Flannery, Teukolsky, \& Vetterling, 1988) to optimize the nonlinear log-likelihood function (Equation A5). The function calculate likelihood is called many times during optimization by Brent_optimization. Note that the log-likelihood function normally requires repeated numerical evaluation of Equation A2. To save time, we precompute $\log \left(p_{k}\right)$ and $\log \left(1-p_{k}\right)$ in Equation A5 as a function of $d^{\prime}$ and store results in a look-up table (LUT). During the optimization procedure, we calculate $d^{\prime}$ from $u, C$, and $D$, and linearly interpolate values from the LUT to compute the likelihood function. (The LUT is very dense and linear interpolation does not lead to significant errors. For 100 responses, we compared

\section{Forced-Choice, Adaptive Contrast}

begin main

constant Dose $=1.0$;

initialize Contrast;

read zero-mean noise from disk into a large real array Noise[];

$u=$ adaptive_contrast(100, Contrast, Dose, Noise[]);

output curves of $u$, uVariance, and Contrast as a function of trial number; end main

adaptive_contrast(MaxTrial, Contrast, Dose, Noise[])

repeat

create_image_sequence(Contrast, Dose, Noise[]);

display image sequence;

(Subject selects one of nine positions with a mouse.)

if subject chooses the disk position, then Correct $=$ true, else Correct $=$ false;

update ResponseData, a triplet (Contrast, Dose, Correct) containing all responses;

* Brent iterative optimization routine calls a user-supplied function, calculate likelihood. */

$u=$ Brent_optimization(calculate_likelihood, ResponseData);

$u$ Var = variance_estimate $($ ResponseData, $u)$;

Contrast $=2.405 /\left(u * \operatorname{Dose}^{1 / 2}\right)$

$I^{*} d^{\prime}=2.405$ for $80 \%$ detectability. $* /$

until (number of trials $\geq$ MaxTrial OR variance is small OR operator terminates); return;

function create_image_sequence(Contrast, Dose, Noise[])

create BackgroundNoise[] from Dose and Noise[]; $\quad$ * Scale and offset appropriately. */

create ObjectNoise[] from Dose, Contrast, and Noise[]; $/ *$ Scale and offset appropriately. */

repeat

repeat

get RandomStart using a random number generator;

/* Create background.*/

blockcopy a pixel row from BackgroundNoise[] starting at RandomStart;

until the noisy image frame is filled;

repeat

get RandomPixel using a random number generator;

copy a pixel from ObjectNoise at RandomPixel;

until the noisy object is filled;

until all frames are created;

return;

function calculate_likelihood(ResponseData)

calculate the log-likelihood function given all values in ResponseData;

${ }^{*}$ Use a look-up table to calculate functions containing numerical integrals. */ return;

Figure B1. The forced-choice, adaptive-contrast algorithm is described in pseudocode. A convention is that function names contain underscores (e.g., create_image_sequence) while variable names are concatenated, capitalized words (e.g., BackgroundNoise). The variable, Contrast, is in bold because it is adapted to maintain an $\mathbf{8 0} \%$ probability of correct detection. (See text for details.) 


\section{Forced-Choice, Adaptive Reference and Test}

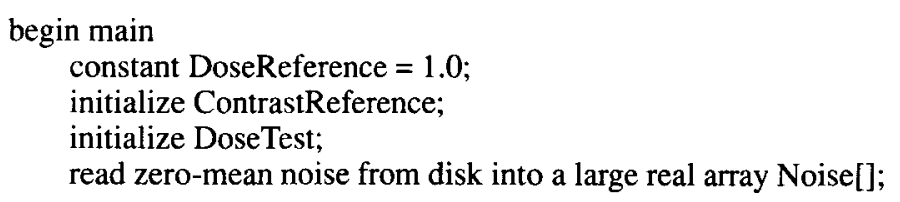

$/ *$ Do 50 reference trials to obtain ContrastReference for $80 \%$ correct. $* /$
uReference $=$ adaptive_contrast $(50$, ContrastReference, DoseReference, Noise[] $)$;

Contrast Test $=$ ContrastReference;

/* Do 50 test trials to obtain DoseTest for $80 \%$ correct. */

uTest $=$ adaptive_dose(50, ContrastTest, DoseTest, Noise[]);

repeat

$1^{*}$ Do a reference trial: create and display sequence, get subject response, */

1* estimate uReference, and compute new ContrastReference. $*$ /

uReference $=$ adaptive_contrast $(1$, ContrastReference, DoseReference, Noise[] $)$;

Contrast $T e s t=$ ContrastReference;

I* Do a test trial: create and display sequence, get subject response, estimate uTest, */

${ }^{*}$ and compute new DoseTest.

uTest $=$ adaptive_dose( 1 , ContrastTest, DoseTest, Noise[]);

until (maximum number of trials reached $O R$ variance is small $O R$ operator terminates);

output uReference, ContrastReference, uTest, and DoseTest as a function of trial number; end main

EquivalentPerceptionDose $=$ DoseTest;

Figure B.2. In the interspersed, forced-choice algorithm, the reference method (pulsed-30) and the test method (pulsed-15) are alternated. In the case of reference, dose is fixed at $1.0 \mathrm{Q} / \mathrm{acq}$ and contrast is adapted to maintain the probability correct at $80 \%$. In the case of test, the contrast is obtained from the last reference display, and dose is adapted to maintain the probability correct at $80 \%$. The final value of DoseTest is the equivalent perception dose (EPD). (See text for details.)

$u$ estimates from our fast interpolation technique with those from direct calculation and found the error in $u$ to be less than $0.01 \%$.)

In our current experiments, the program has taken approximately $5 \mathrm{sec}$ to estimate parameters and create an image sequence display consisting of 100 frames of $384 \times 384$ pixels. Approximately $95 \%$ of this time is spent in create_image_sequence.

In the case of an adaptive-dose experiment, we fix contrast and change dose. The algorithm is very similar except that each time a new image sequence is created, the noise must be scaled appropriately. It takes an additional $2 \mathrm{sec}$ to update the image sequence.

\section{Reference/Test Algorithm}

To control for changes in subject attention, we intersperse reference and test displays using the program described in Figure B.2. We are interested in dose, contrast, and $u$ values for both the reference and test methods. DoseReference is always set at 1.0 Q/acq, giving the nominal noise level described in the Method section. We enter initial values for ContrastReference and DoseTest, and the program updates values over the course of the experiment. To refine the reference estimates, the adaptive-contrast technique is used in initial trials (typically 50 ) to get a good value of ContrastReference for $80 \%$ correct. We next apply 50 test trials. In this case, we fix contrast at the last reference value and adapt dose to find DoseTest for $80 \%$ correct. The test display is linked to reference and always uses the same contrast as in the previous reference presentation.

We now begin the main loop. The program calls adaptive contrast for a single iteration with ContrastReference and the constant, DoseReference. Recall that this function creates an appropriate image sequence, gets the subject response from a mouse input, computes a maximum-likelihood estimate of $u$ Reference using all previous responses, computes a new ContrastReference, and returns control to the main loop. Contrast Test is equated to ContrastReference to ensure that the test display has the same contrast as the reference display. The program next calls adaptive_dose for a single iteration. Upon return, we have new $u$ Test and DoseTest values. The program loops, alternating reference and test displays. The method continues until the stopping criterion (normally a maximum number of presentations) is reached. The final value of DoseTest is the EPD.

(Manuscript received July 11, 1995; revision accepted for publication October $21,1996$. 